HUMAN GENE THERAPY 29:A1-A20 (November 2018)

(C) Mary Ann Liebert, Inc.

DOI: 10.1089/hum.2018.29071.abstracts

\title{
International Conference on Lymphocyte Engineering September 13-15, 2018 Madrid, Spain
}




\section{ICLE 2018 - Oral Presentations}

\section{ICLE8-0039}

Engineering HSC, NK, NKT and $\gamma \delta$ T cells

\section{NK CELLS FROM DIFFERENT SOURCES AS A PROMISING ALTERNATIVE FOR CAR-BASED IMMUNOTHERAPY AGAINST HEMATOLOGICAL CANCERS}

L. Herrera ${ }^{1}$, A. Boronat ${ }^{2}$, P. Zuñiga ${ }^{1}$, I. Martin ${ }^{3}$, J. Anguita ${ }^{3}$, B. Marzal ${ }^{2}$, S. Santos ${ }^{1}$, M.A. Vesga ${ }^{1}$, F. Borrego ${ }^{4}$, M. Juan ${ }^{2}$, C. Eguizabal ${ }^{1}$

${ }^{1}$ Basque Center for Blood Transfusion and Human Tissues, Cell Therapy and Stem Cells Group, Galdakao- 48960- Spain, Spain ${ }^{2}$ Hospital Clínic - Sant Joan de Déu- UB-IDIBAPS,

Immunotherapy Section. Servei d'Immunologia, 08036 Barcelona, Spain ${ }^{3}$ CIC bioGUNE, CIC bioGUNE, Derio48160- Spain, Spain ${ }^{4}$ Basque Center for Blood Transfusion and Human Tissues, Research Unit, Galdakao- 48960- Spain, Spain

Chimeric antigen receptors (CARs) have been successfully used in order to direct autologous $\mathrm{T}$ cells against tumor cells, such as malignant $\mathrm{B}$ cells in acute lymphoblastic leukemia, with encouraging results. A potential problem with this new treatment could be the need of using allogenic $\mathrm{T}$ cells when is not possible to perform an apheresis to the patient, as allogenic $\mathrm{T}$ cells carry a risk of graft versus-host disease (GVHD). Natural killer (NK) cells are part of the innate immune system and they fight against neoplastic cells without previous sensitization. Moreover, allogenic NK cells exhibit a potent graft versus leukemia (GVL) effect without causing GVHD. Because of these characteristics, allogenic NK cells from adult peripheral blood $(\mathrm{AB})$ and cord blood $(\mathrm{CB})$ are an attractive source for cancer immunotherapy. We transduced AB NK cells and $\mathrm{CB}$ NK cells with a lentiviral vector containing CARCD19 with similar transduction efficiency (60-65\%). Moreover, we performed functional assays, including degranulation and cytotoxicity, against hematological cancer cell lines and primary cells from CLL patients that express CD19 cell surface receptor, with the purpose of demonstrating the specific killing efficiency of these CAR-CD19 transduced NK cells. They kill significantly better CD19 expressing target cells than those which are not transduced with CAR-CD19, improving up to 7 times the killing efficiency against primary cells from CLL patients.

In summary, $\mathrm{AB}$ NK cells and $\mathrm{CB}$ NK cells are a promising cell sources to consider for CAR-based immunotherapy, due to the low risk of GVHD.

\section{ICLE8-0047 \\ Genome editing for allogeneic ACT \\ GENOME EDITING USING ARCUS HOMING ENDONUCLEASES AND AN OPTIMIZED MANUFACTURING PROCESS FOR PRODUCTION OF OFF-THE-SHELF ALLOGENEIC CAR T THERAPIES}

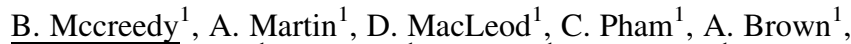
J.A. Hux ${ }^{1}$, J. Lape ${ }^{1}$, C. Beard ${ }^{1}$, J. Smith ${ }^{1}$, K. Nguyen ${ }^{1}$, V. Senyukov ${ }^{1}$, J. Anthony ${ }^{1}$, K. Sivarajan ${ }^{1}$, L. Som ${ }^{1}$, M. Triggiano ${ }^{1}$, D. Jant $z^{1}$

${ }^{1}$ Precision BioSciences, Inc., Cell Therapy, Durham, USA

We have developed a one-step genetic engineering and an optimized manufacturing process for large scale production of allogeneic CAR T cells that are shipped as sterile product in vials and can be stored frozen until needed as off-the shelf ACT. Healthy donor T cells are gene edited using an engineered ARCUS homing endonuclease coupled with transduction using an AAV vector carrying a homology-directed repair template to direct insertion of a CAR transgene into the TRAC locus. This process yields TCR knock-out, CAR knock-in T cells that express a consistent and tunable level of CAR on the cell surface. Our first allogeneic CAR T product, PBCAR0191, is a TCR knock out, targeted knock in of a second generation anti-CD19 CAR with a novel co-stimulatory domain that should enter Phase I clinical testing later this year. We have optimized a large scale, GMP compliant manufacturing process that consistently yields $>1.5 \times 10^{10}$ TCR- CAR+ (PBCAR0191) T cells. The CAR T drug product consists of CD4 and CD8 T cells of which the majority are Tscm and Tcm phenotypes. PBCAR0191 CAR T cells are polyfunctional in terms of cytokine/chemokine production upon co-culture with CD19+ target cells and potently kill CD19+ human tumor cells in vitro. PBCAR0191 CAR $T$ cells showed potent in vivo antitumor activity in immunodeficient mice bearing human ALL (Nalm-6) and NHL (Raji) tumor xenografts resulting in significantly prolonged survival. In a xenogeneic model of GvHD, administration of high doses of PBCAR0191 CAR T cells did not result in GvHD or any other clinically observable toxicities. No alloreactivity was observed for PBCAR0191 CAR T cells in human mixed lymphocyte reaction cultures. Next generation allogeneic CAR $\mathrm{T}$ cells incorporating additional gene edits designed to reduce immunogenicity and resist immunosuppression within the TME are being produced and evaluated in preclinical studies. 


\section{ICLE8-0025}

Genome editing for allogeneic ACT

\section{GENERATION OF “OFF-THE SHELF" $\alpha$ CD19-CAR T CELLS FOR THE TREATMENT OF B CELL MALIGNANCIES}

M. Tristán-Manzano ${ }^{1}$, R. Martín-Guerra ${ }^{1}$, N. Maldonado ${ }^{1}$, S. Sánchez-Hernández ${ }^{1}$, M. Cortijo ${ }^{1}$, C. Marañon ${ }^{1}$, C. Herrera ${ }^{2}$, M.D. Carmona ${ }^{2}$, J.A. Marchal $^{3}$, K. Benabdellah ${ }^{1}$, F. Martín ${ }^{1}$

${ }^{1}$ Centro Pfizer - Universidad de Granada - Junta de Andalucía de Genómica e Investigación Oncológica GENYO-, Genomic Medicine, Granada, Spain ${ }^{2}$ Unidad de Terapia Celular IMIBICHURS, Hospital Reina Sofía, Córdoba, Spain ${ }^{3}$ Centre for Biomedical Research CIBM-University of Granada, Human Anatomy and Embryology, Granada, Spain

Current adoptive immunotherapy strategies use autologous $\mathrm{T}$ cells expressing a Chimeric Antigen Receptor (CAR) against CD19+ B cells malignancies. Beyond the impressive benefit, time and quality of manufacturing $\mathrm{T}$ cells from the refractory patients constitutes a critical limitation for a successful therapy. Allogenic universal CAR-T cells can overcome the histocompatibility problem and can be generated from healthy donors to treat several patients. Targeting TCR can avoid graft versus host disease (GVHD) and allow the preservation of a less exhausted CAR-T cells population. In this line, we have constructed LVs expressing a third generation $\alpha \mathrm{CD} 19-\mathrm{CAR}$ and modified efficiently human primary T cells. $\alpha$ CD19-CAR-T cells showed good efficacy and selectivity by destroying only CD19+ cells and achieving more than $80 \%$ specific lysis of a Burkitt-lymphoma cell line after only $24 \mathrm{~h}$ of co-culture. Moreover, we have generated primary T cells that are 70-90\% deficient for the TCR using nucleofection of Cas9/gRNA ribonucleoproteins. TCRKO T cells showed a higher expression of naïve and memory CD45RA and CD62L markers. Finally, we have obtained primary T cells that were $80 \%$ TCRKO and $90 \%$ positive for $\alpha \mathrm{CD} 19-\mathrm{CAR}$. At this point, we are monitoring phenotypic changes and studying the efficacy and safety of these TCRKO- $\alpha$ CD19CAR T cells to eradicate CD19 positive cells in vitro and in vivo.

\section{ICLE8-0045}

HIV

\section{ANTIBODY-SECRETING T CELLS ENGINEERED FOR TRIPARTATE IMMUNE RESPONSE AGAINST THE HIV RESERVOIR}

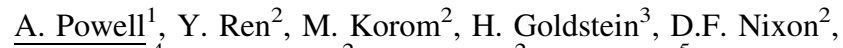

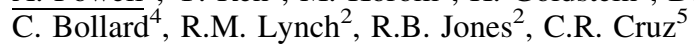

${ }^{1}$ George Washington University, Institute for Biomedical Sciences, Washington, DC, USA ${ }^{2}$ George Washington University, Institute for Microbiology-Immunology- and Tropical Medicine, Washington, DC, USA ${ }^{3}$ Albert Einstein College of Medicine, Department of Microbiology and Immunology, New York, New York, USA ${ }^{4}$ Children's National Medical Center, Center for Cancer \& Immunology Research, Washington, DC, USA ${ }^{5}$ Children's National Medical Center, Department of Pediatrics, Washington, DC, USA

Antiretroviral therapies have improved outcomes in HIV/ AIDS patients but are unable to cure infection, because they are unable to target the reservoir which evades immunity and re-seeds infection. Several strategies appear promising: (1) neutralizing antibodies from patient sera prevent virus from in- fecting cells, (2) strong $\mathrm{T}$ cell responses seen in patients with low viral loads, and (3) subjects vaccinated in the RV144 study have high levels of ADCC. But the simultaneous use of all three as the basis of a single therapeutic has never been explored. We sought to modify HIV-specific T cells from HIV-naïve donors (dHXTC, to allow use in the context of allotransplant, a promising platform for sterilizing cures) to express a neutralizing antibody directed against HIV envelope (10-1074).

We designed a 10-1074 construct comprised of 10-1074 heavy and light chains fused to IgG3 Fc to elicit ADCC, with truncated CD19 as selectable marker. HIV-specific T cells were expanded from HIV-naïve donors by priming with antigen presenting cells expressing overlapping HIV antigens in the presence of cytokines.

$\mathrm{T}$ cells retained specificity against gag, nef, and pol peptides $\left(89.568 \pm 71.093 \mathrm{INFg}\right.$ SFC $/ 1 \times 10^{5}$ cells against HIV peptide vs $32.7 \pm 37.869 \mathrm{INFg} \mathrm{SFC} / 1 \times 10^{5}$ cells against irrelevant peptide, $\mathrm{n}=5)$ following transduction $(30.18 \pm 15.39 \%, \mathrm{n}=5)$ with the 10 1074 construct. These cells secreted 10-1074 antibodies (144.2 \pm $77.12 \mathrm{ng} / \mathrm{mL}, \mathrm{n}=5, \mathrm{p}=0.0139$ compared to untransduced cells). Functional analysis of the secreted engineered 10-1074 bnAb in culture supernatants showed higher neutralization compared to their untransduced counterparts $(39.81 \pm 17.16 \%$ compared to $6.76 \pm 1.25 \%$ in untransduced) of HIV and ADCC as shown by NK cytotoxicity (10.6\%) in 2/3 dHXTC products tested.

We conclude that HIV-specific T cells can be engineered to elicit ADCC against HIV envelope and neutralize HIV. This tripartite approach allows for synergy between immune arms, broadens the target range of the immune therapy, and provides further insight into what defines an effective anti-HIV response.

\section{ICLE8-0023}

Non-viral vectors and transposons

\section{CRISPR/CAS9 UNITES WITH SLEEPING BEAUTY TO GENERATE CAR T CELLS WITH ENHANCED THERAPEUTIC INDEX FOR FIGHTING AGAINST IMMUNOSUPPRESSIVE TUMOR MICROENVIRONMENT}

R. Monjezi ${ }^{1}$, C. Miskey ${ }^{2}$, T. Stüber ${ }^{3}$, E. Grueso ${ }^{4}$, M. Schleef ${ }^{5}$, M. Schmeer ${ }^{5}$, H. Einsele ${ }^{1}$, Z. Ivics ${ }^{2}$, M. Hudecek ${ }^{1}$

${ }^{1}$ universitätsklinikum würzburg, Internal medizinische klinik und poliklinik ii, Würzburg, Germany ${ }^{2}$ Paul-Ehrlich-Institute, Division of Medical Biotechnology, Langen, Germany ${ }^{3}$ universitätsklinikum würzburg, Frauenklinik und Poliklinik, Würzburg, Germany ${ }^{4}$ Universidad Francisco de Vitoria, Department of Biotechnology, Pozuelo de Alarcón, Spain ${ }^{5}$ PlasmidFactory, PlasmidFactory, Bielefeld, Germany

Adoptive immunotherapy with CD19-specific chimeric antigen receptor (CAR) is effective for treating B-cell malignancies. However, extrapolating this paradigm to other hematologic and prevalent solid tumors requires more advanced bioengineering of CAR T cells and likely, the ability for concomitant genome editing to augment their therapeutic potency. Therefore, we developed a platform that enables simultaneous and virus-free Sleeping Beauty (SB) gene transfer and CRISPR/Cas9 genome editing in $\mathrm{T}$ cells to create genome-edited CAR $\mathrm{T}$ cells with improved antitumor function.

We generated CAR T cells specific for CD19 (lymphoma), SLAMF7 (multiple myeloma) and ROR1 (breast cancer) tumor antigens being edited at $T G F \beta-R I I$ or $P D-1$ locus to abrogate the 
signaling mediated by these receptors which leads to impaired T-cell function within tumor microenvironment.

A high rate of CAR expression $(\approx 40 \%, \mathrm{n}=6)$ and knockout (TGF $\beta-R I I \approx 50 \%, P D-1 \approx 80 \%, \mathrm{n}=6$ ) with low toxicity was achieved which enabled rapid $\mathrm{T}$-cell expansion to therapeutic doses. High-throughput sequencing revealed random SB CAR transposition in the T-cell genome, including a high rate of genomic safe harbor integrations, and no guide RNA off-target activity. Compared with non-edited CAR T cells, TGF $\beta$-RII- or PD-1-CAR T cells conferred more potent antitumor functions against cancer cell lines in vitro, in the presence of exogenous $\operatorname{TGF} \beta$ or the target cells that we engineered to overexpress PD-L1, respectively. Experiments that demonstrate enhanced therapeutic efficacy of the CAR T cells in murine xenograft models are currently completing follow-up.

Collectively, we developed a novel T-cell engineering platform to accomplish concomitant CAR gene transfer and genome editing in a single-step and virus-free manner. Genome-edited CAR $T$ cells possess enhanced therapeutic potential in our preclinical analyses and a safe genomic transposon integration and genome editing profile. Manufacturing is currently adopted under GMP-conditions in preparation for clinical trials that evaluate the safety and efficacy of these advanced CAR T-cell products.

\section{ICLE8-0015}

Non-viral vectors and transposons

VAPOR NANOBUBBLE PHOTOPORATION AS A NEW, SAFE AND EFFICIENT TECHNIQUE FOR THE DELIVERY OF SMALL INTERFERING RNA TO CYTOTOXIC T CELLS

\author{
J. Van Hoeck $^{1}$, L. Wayteck ${ }^{1}$, R. Xiong ${ }^{1}$, K. Braeckmans ${ }^{1}$, \\ S.C. De Smedt ${ }^{1}$, K. Raemdonck ${ }^{1}$ \\ ${ }^{1}$ Ghent University, Faculty of Pharmaceutical Sciences, Ghent, \\ Belgium
}

The success of cancer immunotherapy through the adoptive transfer of cytotoxic T lymphocytes (CTLs) is highly dependent on the potency of the elicited anti-tumor responses generated by the transferred cells, which can be hindered by a variety of upregulated immunosuppressive pathways. Downregulation of these pathways in the $\mathrm{T}$ cells via RNAinterference (RNAi) could significantly boost their capacity to infiltrate tumors, proliferate, persist, and eradicate tumor cells, thus leading to a durable anti-tumor response. Unfortunately, it is well known that primary $\mathrm{T}$ cells are hard-to-transfect and conventional nonviral transfection agents are generally ineffective. Viral transduction and electroporation are more efficient but their use is restricted by high cost, safety issues, and cytotoxicity. Photoporation has recently gained interest as a more gentle alternative physical approach to deliver membrane-impermeable macromolecules into cells. By attaching gold nanoparticles (AuNPs) to the cell surface followed by pulsed laser illumination, transient membrane pores can be generated that allow the diffusion of macromolecules directly into the cell cytosol. Here, we evaluated this technique for the non-toxic and effective delivery of small interfering RNA (siRNA) and subsequent silencing of target genes in activated CTLs and Jurkat E6.1 cells. We compared photoporation with nucleofection, the current standard physical technique for $\mathrm{T}$ cell transfection, and demonstrated a significantly reduced cytotoxicity and higher average dose per cell for the photoporation technique.

\section{ICLE8-0031}

Other

EVALUATION OF A FULLY HUMAN CAR FOR THE TREATMENT OF HEPATITIS B VIRUS INFECTION IN AN IMMUNOCOMPETENT MOUSE MODEL

M. Festag $^{1}$, K. Wisskirchen ${ }^{1,2}$, J. Hasreiter ${ }^{1}$, S. Schreiber ${ }^{1}$, H. Abken $^{3}$, U. Protzer ${ }^{1,2}$

${ }^{1}$ Institute of Virology, Technische Universität München/ Helmholtz Zentrum München, Munich, Germany ${ }^{2}$ German Centre for Infection Research DZIF, Munich partner site, Munich, Germany ${ }^{3}$ Center for Molecular Medicine Cologne, University of Cologne, Cologne, Germany

Adoptive T-cell therapy utilizing CARs to redirect $\mathrm{T}$ cells is a promising approach to treat various cancers and infections. Immunocompetent mouse models are needed to obtain a sophisticated understanding of the therapeutic potential and side-effects to expect in clinical application. Still, the evaluation in immunocompetent preclinical models is limited by the possible immune response against human-derived domains of CAR constructs. Here we present an approach to study a fully human CAR in a syngenic immunocompetent mouse model. The CAR is specific for the hepatitis $B$ virus $(\mathrm{HBV})$ surface antigen expressed on HBV-infected hepatocytes and HBV-associated hepatocellular carcinoma. The CAR and the co-expressed safe-guard mechanism truncated epidermal growth factor receptor (EGFRt) induced both CD8 $\mathrm{T}$ cell and antibody responses in immunocompetent mice that led to a rapid elimination of CAR T cells and a limited antiviral effect. First, we demonstrated how to easily test mouse models for an immune response against these kinds of molecules. Following, we circumvented the immune response and induced tolerance to the human-derived domains by total body irradiation (TBI) and a subsequent transfer of nonfunctional CAR T cells. If functional CAR T cells were transferred at a later time point after the endogenous immune system had recovered from TBI, CAR T cell exhibited enhanced engraftment and persisted in high numbers for more than three months. CAR T-cell persistence in tolerized mice permitted a pronounced lasting antiviral effect without inducing intolerable side effects. The presented mouse model will allow the study of other CARs with human-derived domains in the context of an intact endogenous immune system. This will allow the investigation of interaction between CAR $\mathrm{T}$ cells and endogenous immune cells and the consequences for efficacy and safety.

\section{ICLE8-0038}

Other

\section{ARMING HUMAN REGULATORY T CELLS FROM HEALTHY DONORS OR MULTIPLE SCLEROSIS PATIENTS WITH SWITCHABLE CHIMERIC ANTIGEN RECEPTORS FOR THE TREATMENT OF INFLAMMATORY DISEASES}

$\underline{\text { A. Kegler }}^{1}$, S. Koristka ${ }^{1}$, R. Bergmann ${ }^{1}$, C. Arndt ${ }^{1}$, A. Feldmann ${ }^{1}$, S. Albert ${ }^{2}$, G. Ehninger ${ }^{3,4,5}$, M. Bornhäuser ${ }^{3,4,5}$, M. Schmitz ${ }^{4,5,6}$, K. Akgün ${ }^{7}$, T. Ziemssen ${ }^{7}$, M. Bachmann ${ }^{1,2,4,5}$

${ }^{1}$ Helmholtz-Zentrum Dresden-Rossendorf HZDR, Institute of Radiopharmaceutical Cancer Research, Dresden, Germany

${ }^{2}$ UniversityCancerCenter UCC 'Carl Gustav Carus' TU Dresden, Tumor Immunology, Dresden, Germany ${ }^{3}$ University Hospital 'Carl Gustav Carus' TU Dresden, Medical Clinic and Policlinic I, Dresden, Germany ${ }^{4}$ National Center for Tumor 
Diseases NCT, 'Carl Gustav Carus' TU Dresden, Dresden, Germany ${ }^{5}$ German Cancer Research Center DKFZ and German Cancer Consortium DKTK, partner site Dresden, Heidelberg, Germany ${ }^{6}$ Medical Faculty TU Dresden, Institute of Immunology, Dresden, Germany ${ }^{7}$ Center of Clinical Neuroscience University Hospital Carl Gustav Carus' TU Dresden, Department of Neurology, Dresden, Germany

Regulatory $\mathrm{T}$ cells (Tregs) possess a central role in impeding harmful immune reactions and represent important immunomodulatory players in the pathogenesis of inflammatory diseases. For that reason, Tregs are intensively studied as an innovative cell product for the treatment of autoimmunity e.g. multiple sclerosis (MS) or Graft-versus-Host disease. In recent years, several preclinical mouse model clearly demonstrate a superior suppressive capacity of antigen-specific Tregs compared to polyclonal cells. On the downside, isolation of Tregs with a distinct antigen-specificity is a highly time-consuming and laborious process.

To overcome these challenges, we armed polyclonal Tregs isolated from healthy donors or MS patients with a universal chimeric antigen receptor (UniCAR) construct. As $\mathrm{T}$ cells and target cells are indirectly cross-linked by a separate, antigenbinding targeting module (TM), this innovative technology enables side-specific redirection of Tregs to any desired surface structure. Moreover, UniCAR-armed Tregs are silenced in the absence of the TM allowing for a finely tuned regulation of Treg activity between an "on" and "off" status.

Here, we demonstrate that highly pure $\mathrm{CD} 44^{+} \mathrm{CD} 25^{\text {high }}$ CD127 $7^{\mathrm{dim}} \mathrm{CD} 45 \mathrm{RA}^{+}$Tregs isolated from both healthy donors or MS patients stably express a UniCAR construct with an intracellular $4-1 \mathrm{BB} / \zeta$ signaling domain. UniCAR-engrafted Tregs vigorously expand and maintain their phenotype even under pro-inflammatory conditions. Most importantly, upon TMactivation UniCAR-endowed Tregs significantly hamper autologous $\mathrm{T}$ effector cells both in vitro and in vivo.

Taken together, our results underline the enormous therapeutic potential of the UniCAR system for treatment of inflammatory diseases including MS, as it facilitates an antigen-specific and precisely controlled Treg activation at the side of inflammation. Moreover, this innovative technology allows redirection of Tregs against a wide range of surface structures simply by exchanging the TM and might thereby broaden current treatment modalities.

\section{ICLE8-0030}

Receptor and vector design

\section{OVERCOMING FRATRICIDE TO DELIVER} A CLINICAL GRADE CAR T CELL PRODUCT

S. Bornschein $^{1}$, B. Demoulin ${ }^{1}$, E. Breman ${ }^{1}$, A. Michaux ${ }^{1}$, J. Houssa $^{1}$, F. Huberty ${ }^{1}$, B. Violle ${ }^{1}$, C. Jacques-Hespel ${ }^{1}$, C. Marchand ${ }^{1}$, J. Marijsse ${ }^{1}$, T. Nguyen ${ }^{1}$, N. Ramelot ${ }^{1}$, D. Daro ${ }^{1}$, V. Steenwinckel ${ }^{2}$, S. Agaugue ${ }^{1}$, D. Gilham ${ }^{1}$

${ }^{1}$ Celyad, Research \& Development, Mont-Saint-Guibert, Belgium ${ }^{2}$ Celyad, Industrialization, Mont-Saint-Guibert, Belgium

The adoptive transfer of chimeric antigen receptor (CAR) T cells provides an attractive approach to putatively target any tumor cell. However, the success of this approach is limited by the availability of tumor-specific antigens. NKG2D is an activating receptor, most commonly expressed by NK cells and activated T cells, with ligand engagement resulting in target cell killing. NKG2D ligands, MICA, MICB and ULBP1-6 are induced by cellular stress, infections or malignant transformation, while being rarely detectable on the surface of healthy cells. This renders NKG2D ligands an attractive target for a CAR T cell therapy, potentially against a broad panel of hematological and solid cancers.

We engineered a NKG2D-based CAR (NKR-2) consisting of a fusion of the full length NKG2D receptor with the intracellular domain of $\mathrm{CD} 3 \mathrm{z}$, while the accessory protein DAP10 provides the co-stimulatory signal. We have shown that human NKR-2 CAR T cells (CYAD-01) specifically recognize and kill cancer cell lines from distinct types of liquid and solid tumors.

However, $\mathrm{T}$ cell stimulation via the CAR or the $\mathrm{T}$ cell receptor complex induced transient up-regulation of the NKG2D ligands MICA and MICB, resulting in self-killing or fratricide during cell expansion. Inclusion of a Phosphoinositol-3-Kinase inhibitor (LY294002) during the manufacturing process reduced NKG2D expression at the cell surface and partially blunted the fratricide effect, providing a first generic means of generating sufficient numbers of CYAD-01 T cells to initiate the THINK clinical trial (NCT03018405). We further developed this process by means of introducing a monoclonal antibody that selectively binds NKG2D and thereby reduces fratricide to negligible levels with a concomitant improvement in cell yields. This modified process is now delivering ample cell numbers for all doses of the THINK clinical trial. These developments provide a potential route to overcome fratricide should it be an issue for CAR T cell manufacture.

\section{ICLE8-0024}

Safety switches and controlled expression

\section{DEVELOPMENT OF CLINICAL-GRADE DOXYCYCLINE-INDUCIBLE CAR T CELLS}

N. Maldonado $^{1}$, M. Tristán-Manzano ${ }^{1}$, S. Sánchez-Hernández ${ }^{1}$, C. Marañón ${ }^{1}$, C. Herrera ${ }^{2}$, M.D. Carmona ${ }^{2}$, F. Martin ${ }^{1}$, K. Benabdellah ${ }^{1}$

${ }^{1}$ Centro Pfizer - Universidad de Granada - Junta de Andalucía de Genómica e Investigación Oncológica GENYO, Genomic Medicine, Granada, Spain ${ }^{2}$ Unidad de Terapia Celular en Hospital Reina Sofía, Servicio Andaluz de Salud, Córdoba, Spain

$\mathrm{T}$ cells genetically modified with a $\alpha \mathrm{CD} 19$ chimeric antigen receptor ( $\alpha$ CD19-CAR) have demonstrated remarkable potency in B cell malignancies in clinical trials. Nonetheless, there are important safety problems such as cytokine release syndrome (CRS), neurologic toxicity (NT), and on-target off-tumor toxicity. To solve these adverse events, we aimed to develop a clinical-grade inducible CAR that would be activated only in the presence of the inductor (doxycycline, Dox). The main drawbacks of inducible systems are the presence of leaking and/or of transactivators (TetR-VP16 chimeras) that can alter the target cells phenotype and are therefore not appropriate for clinical applications. We have previously generated all-in-one LVs (LentOnPlus), based on the original TetR repressor, that achieve very low leaking and good inducibility in most target cells. In this work we have analyzed the behavior of LentOnPlus systems, containing or not the Is 2 insulator, in Jurkat and primary $\mathrm{T}$ cells. Both LVs generated Dox-responsive Jurkat cells with low leaking and high inducibility. However, only with the LentOnPlus LVs harboring the Is 2 insulator generated primary T cells that responded to doxycycline. Interestingly, all the different $\mathrm{T}$ cells subpopulations (central memory, naïve/stem and effectors) responded similarly to the regulator. We next replace eGFP with a thirdgeneration $\alpha \mathrm{CD} 19-\mathrm{CAR}$ in both LentOnPlus backbones to generate CCARET and CCARETIs2. Again only the CCARETIs2 generated inducible primary $\mathrm{T}$ cells expressing $\alpha \mathrm{CD} 19-\mathrm{CAR}$ only in the presence of doxycycline. Ongoing experiments aim to demonstrate the Dox-dependant in vitro and in vivo CD19-specific killing activity of these inducible $\mathrm{T}$ cells. 


\section{ICLE8-0040}

Solid tumours

\section{REDIRECTING SWITCHABLE UNICAR T CELLS FOR ELIMINATION OF RADIORESISTANT CANCER CELLS}

\author{
A. Feldmann ${ }^{1}$, C. Arndt ${ }^{1}$, R. Bergmann ${ }^{1}$, N. Berndt ${ }^{2}$, \\ J. Jureczek ${ }^{2}$, S. Albert $^{3}$, D. Lindner ${ }^{3}$, S. Koristka ${ }^{1}$, \\ J. Steinbach ${ }^{1,2}$ G. Ehninger ${ }^{2,4}$, M. Krause ${ }^{2,5,6}$, I. Kurth ${ }^{5}$, \\ A. Dubrovska ${ }^{2,5,6}$, M. Bachmann ${ }^{1,2,3}$ \\ ${ }^{1}$ Helmholtz-Zentrum Dresden-Rossendorf HZDR, Institute of \\ Radiopharmaceutical Cancer Research, Dresden, Germany \\ ${ }^{2}$ German Cancer Research Center DKFZ and German Cancer \\ Consortium DKTK, partner site Dresden, Heidelberg, Germany \\ ${ }^{3}$ University Cancer Center UCC 'Carl Gustav Carus' TU Dresden, \\ Tumor Immunology, Dresden, Germany ${ }^{4}$ University Hospital \\ 'Carl Gustav Carus' TU Dresden, Medical Clinic and Policlinic I, \\ Dresden, Germany ${ }^{5}$ Medical Faculty TU Dresden, OncoRay - \\ National Center for Radiation Research in Oncology, Dresden, \\ Germany ${ }^{6}$ Helmholtz-Zentrum Dresden-Rossendorf HZDR, \\ Institute of Radiooncology - OncoRay, Dresden, Germany
}

Radiation therapy represents a commonly applied treatment regimen for solid tumors. Unfortunately, it is often accompanied by a high risk for the outgrowth of radioresistant cancer cells against which treatment options are limited. We challenged the idea whether or not chimeric antigen receptor (CAR)-modified $\mathrm{T}$ cells could be exploited as an adjuvant immunotherapy in combination with standard radiotherapy. Over the past several years, we have established switchable universal CAR constructs (UniCARs) that recognize a short peptide epitope (E5B9) which does not exist on the surface of living cells. UniCAR $\mathrm{T}$ cells are redirected to malignant cells exclusively in the presence of a target module (TM) that contains the epitope E5B9 and specifically binds to a tumor-associated antigen (TAA) on the tumor cell surface.

For providing a rationale for the combination of CAR and radiation therapy, we used different radioresistant sublines of the head and neck cancer cell line Cal33. Expression of various TAAs including of PSCA, EGFR and CD98 was confirmed by flow cytometry analysis. Subsequently, TMs recognizing these potential targets were generated from the variable domains of monoclonal antibodies, cloned into lentiviral vectors and purified from cell culture supernatants of TMproducing stable cell lines. In parallel, T cells isolated from healthy donors were engrafted with UniCARs by lentiviral transduction. Armed with our anti-TAA TMs, UniCAR T cells efficiently lysed radioresistant $\mathrm{Cal} 33$ tumor cells both in vitro and in vivo.

Taken together, we could demonstrate that radioresistant cancer cells can effectively be killed by retargeting UniCAR $\mathrm{T}$ cells against PSCA, CD98 and EGFR. Thus, resistance to standard of care radiotherapy can be overcome by concomitant or subsequent immunotherapy using the flexible UniCAR technology.

\section{ICLE8-0021}

Solid tumours

\section{CHIMERIC ANTIGEN RECEPTORS WITH A MYD88 AND CD40 ENDODOMAIN ENDOW T CELLS WITH SUPERIOR ANTITUMOR ACTIVITY}

\section{B. Prinzing $^{1}$, G. Krenciute ${ }^{1}$, S. Gottschalk ${ }^{1}$ \\ ${ }^{1}$ St. Jude Children's Research Hospital, Bone Marrow \\ Transplantation \& Cellular Therapy, Memphis, USA}

Adoptive cell therapy with T-cells expressing chimeric antigen receptors (CARs) has not yet been nearly as successful for solid tumors as for leukemia. Lack of efficacy is most likely due to several factors, including suboptimal CAR signaling resulting in limited Tcell activation within the solid tumor microenvironment. The majority of CARs encode an endodomain that consists of zeta $(\mathrm{z})$ and a canonical CD28 and/or 41BB costimulatory signaling domain. However, other molecules play important roles in T-cell costimulation, and we are evaluating the use of the TLR adaptor molecule MyD88 and the TNFR CD40 molecule as CAR endodomains.

We generated CARs with CD28.z, 41BB.z, or MyD88.CD40.z endodomains that recognize the solid tumor antigen EphA2. In coculture assays with EphA2+ tumor cells, CAR.CD28.z T-cells killed faster and secreted significantly more Th1 cytokines than CAR.MyD88.CD40.z or CAR.41BB.z, which produced cytokines and killed at similar rates. Consistent with greater T-cell activation, CAR.CD28.z T-cell cultures had a higher percentage of terminally differentiated $\mathrm{T}_{\mathrm{EMRA}}$ cells (CD45RA+/CCR7-) in comparison to CAR.MyD88.CD40.z T cells. In sequential killing assays only CAR.MyD88.CD40.z T-cells maintained their ability to expand and kill tumor cells in contrast to CAR.CD28.z or CAR.41BB.z T-cells.

In vivo, we have so far compared the expansion, persistence, and anti-tumor activity of MyD88.CD40.z and 41BB.z CAR Tcells in the EphA2+ LM7 osteosarcoma xenograft model. MyD88.CD40.z CAR T-cells had greater antitumor activity than 41BB.z CAR T-cells, resulting in a significant survival advantage. In addition, MyD88.CD40.z CAR T-cells expanded more and persisted longer than 41BB CAR T-cells, providing a potential mechanism for their improved anti-tumor activity.

Our results suggest that CAR.MyD88.CD40.z T-cells have greater antitumor activity in vitro and in vivo in comparison to CAR.CD28.z or 41BB.z T-cells. Thus, including MyD88 and CD40 signaling domains in CAR T-cells may improve current CAR T-cell therapy approaches for solid tumors.

\section{ICLE8-0016}

Target and receptor discovery

\section{HARNESSING CD8 CO-RECEPTOR FUNCTION FOR IMMUNOTHERAPY WITH LOW-AVIDITY TCR TRANSGENIC T CELLS}

G. Bajwa ${ }^{1}$, E. Hoyer ${ }^{1}$, D. Brenner ${ }^{1}$, M. Martinez-Paniagua ${ }^{2}$, N. Nouraee ${ }^{1}$, F. Sadeghi ${ }^{2}$, I. Gruber ${ }^{3}$, M. Hebeisen ${ }^{3}$, N. Rufer ${ }^{3}$, M.F. Wu ${ }^{4}$, W. Tao ${ }^{4}$, N. Varadarajan ${ }^{2}$, M. Brenner ${ }^{1}, \underline{\text { C. Arber }}{ }^{3}$

${ }^{1}$ Baylor College of Medicine, Center for Cell and Gene Therapy, Houston, USA ${ }^{2}$ University of Houston, Department of Chemical and Biomolecular Engineering, Houston, USA ${ }^{3}$ Ludwig Institute for Cancer Research Lausanne-Lausanne University Hospital and University of Lausanne, Department of oncology UNIL CHUV, Lausanne, Switzerland ${ }^{4}$ Baylor College of Medicine, Department of Biostatistics, Houston, USA

Most naturally occurring major histocompatibility class I restricted $\mathrm{T}$ cell receptors (TCRs) that target over-expressed tumorassociated self-antigens (TAAs) are of low avidity due to selection and tolerance in the host and are CD8 co-receptor dependent. Adoptive $\mathrm{T}$ cell transfers with TCR-engineered $\mathrm{T}$ cells thus rely on the function of CD8+ T cells and do not exploit beneficial $\mathrm{CD} 4+\mathrm{T}$ cell functions. Hence, we developed a novel strategy that combines expression of a TAA-specific low-avidity TCR with the $\mathrm{CD} 8 \alpha \beta$ co-receptor and explored the properties of purified transgenic CD8+ and CD4+ T cells separately, in vitro and in vivo. We found that $\mathrm{CD} 8 \alpha \beta$ co-transfer enhanced TCR+ CD8+ T cell function by increasing their serial tumor killing capacity, indicating that limited availability of endogenous CD8 co-receptors impedes full deployment of their functional potential. Engineered CD4+ $\mathrm{T}$ cells were efficiently reprogrammed into hybrid multifunctional cytotoxic and helper T cells at the single cell level: they 
recognized and killed cells expressing the cognate class I restricted tumor antigen, became serial killers, produced mostly $T_{H} 1$ and preserved some $\mathrm{T}_{\mathrm{H}} 2$ cytokines, and showed superior antitumor function in vivo in a leukemia xenograft model. $\mathrm{CD} 8 \alpha \beta$ cotransfer restored the TCR-pMHC interaction in CD4+ T cells and enhanced early TCR signaling events. In conclusion, we present a novel approach that allows us to (1) enhance the function of TCRtransgenic CD8+ T cells and (2) to manufacture class I pMHC targeted hybrid cytotoxic and helper T cells with both CD8+ and $\mathrm{CD} 4+\mathrm{T}$ cell functions readily available at the single cell level.

\section{ICLE8-0028}

Target and receptor discovery

\section{A SEMI-AUTOMATED METHOD FOR THE ISOLATION AND INITIAL CHARACTERIZATION OF} NEOANTIGEN-SPECIFIC T CELL RECEPTORS

\section{A. Bracher $^{1}$, D. Sommermeyer ${ }^{1}$ \\ ${ }^{1}$ Medigene Immunotherapies $\mathrm{GmbH}, \mathrm{T}$ Cell Engineering, Planegg-Martinsried, Germany}

Adoptive $\mathrm{T}$ cell therapy using tumor-infiltrating lymphocytes (TILs) seems to be most successful if the TILs that are expanded ex vivo and reinfused into patients include $\mathrm{T}$ cells specific for individual tumor mutations. In addition, the success of checkpoint inhibitors is correlated with the mutational load of tumors indicating that responding tumors carry TILs specific for mutated epitopes displayed by the tumor cells. Targeting neoantigens for patient-individualized therapy encompasses a number of hurdles. The high number of mutations as potential targets presents the necessity to have in place robust processes for rational, rapid selection and validation of neoantigens as $\mathrm{T}$ cell targets. In addition, the assessment of safety becomes paramount given that neoepitopes may differ by only one residue from the normal cellular protein. Multiple immunotherapy platform technologies were used to investigate neoantigens as future targets for adoptive $\mathrm{T}$ cell therapies. In a proof of concept experiment targeting seven different neoantigens in vitro, $T$ cell responses against four of these antigens from peripheral $\mathrm{T}$ cells of healthy donors were detected. Using an automated $\mathrm{T}$ cell screening platform, a variety of neoantigen-specific $\mathrm{T}$ cell clones were isolated and subsequently the $\mathrm{T}$ cell receptor (TCR) sequences were identified by targeted Next Generation Sequencing (NGS). After retroviral transfer of the TCR sequences, donor T cells were able to specifically recognize neoantigen-expressing target cells, release cytokines and show potent cytotoxicity.

\section{ICLE 2018 - ePoster Presentations}

\section{ICLE8-0026}

Hematological malignancies

\section{LYMPHOCYTE-SPECIFIC DELIVERY OF PROTEIN THERAPEUTICS BY GENETICALLY ENGINEERED EXOSOMES}

\author{
D. Levy ${ }^{1}$, M. Do ${ }^{1}, \underline{B . ~ L u}^{1}$ \\ ${ }^{1}$ Santa Clara University, Department of Bioengineering, \\ Santa Clara, USA
}

Targeted and intracellular delivery of therapeutic proteins to specific cell types represents a promising technology for the potential treatment of various human diseases. Recently, cellderived nano-vesicles termed exosomes have emerged as a robust tool for both in vitro and in vivo delivery of therapeutic nucleic acids. Here we present a new strategy for targeted delivery of bioactive proteins via genetically engineered exosomes. By combining a CD19-specific binding receptor with an exosomal transmembrane scaffold and a green fluorescent protein, we were able to effectively load targeted peptides as well as reporter proteins onto the membrane surface and lumen of exosomes respectively. We demonstrated that genetically transformed human cells could produce these engineered exosomes indefinitely. Characterization of the isolated exosomes showed that they were properly modified. Uptake experiments exhibited that engineered exosomes could deliver bioactive cargo proteins inside targeted cells. This technology provides a new tool for targeted delivery of potential protein therapeutics to
CD19-positive lymphocytes. This strategy paves a new path for the targeted treatment of blood malignancies, including B-cell leukemia or lymphoma.

\section{ICLE8-0007}

Hematological malignancies

\section{SUPER-RESOLUTION MICROSCOPY DSTORM REVEALS CD19DIM EXPRESSION ON A SUBSET OF MYELOMA CELLS THAT CAN BE TARGETED WITH CD19-CAR T CELLS}

T. Nerreter $^{1}$, S. Letschert ${ }^{2}$, S. Doose ${ }^{2}$, S. Danhof ${ }^{1}$, H. Einsele ${ }^{1}$, M. Sauer ${ }^{2}$, M. Hudecek ${ }^{1}$

${ }^{1}$ University Hospital of Wuerzburg, Medicine II, Wuerzburg,

Germany ${ }^{2}$ Julius-Maximilians-University Wuerzburg,

Department of Biotechnology and Biophysics, Wuerzburg, Germany

Background: Since the inception of chimeric antigen receptor (CAR) development, there has been controversy over the amount of target molecules required to activate $\mathrm{T}$ cells via the CAR. Here, we evaluate the use of CAR-engineered T-cells targeting CD19 (CD19CART) in multiple myeloma, a clonal proliferation of plasma cells. A recent study (Garfall et al, NEJM 2015) reported complete remission in a patient that had received CD19CART even though only $0.05 \%$ of myeloma cells expressed CD19 as judged by flow cytometry (FC). This has sparked debate over low 
level CD19 expression on myeloma cells that may not be detectable by FC but trigger elimination by CD19CART.

Methods: We generated expression profiles of CD19 on myeloma cells from $n=14$ patients by single-molecule sensitive super-resolution microscopy ( $d$ STORM - direct stochastic optical reconstruction microscopy) and FC. In parallel, we treated myeloma cells with CD19CART and control T cells in vitro.

Results: In 10/14 patients, we detected CD19 on a fraction of myeloma cells (range: $10.3 \%-80 \%$ ) by $d$ STORM, while FC detected CD19 only in 2/14 patients on a smaller cell fraction (range: 4.9\%-30.4\%). Four patients were classified as CD19-negative by $d$ STORM. The majority of myeloma cells expressed CD19 at very low levels, far below the FC detection limit. Treatment with CD19CART led to specific elimination of CD19 ${ }^{\text {dim }}$ myeloma cells, even when CD19 was undetectable by FC.

Conclusions: In a prevailing subset of patients, CD19 is expressed on a large fraction of myeloma cells at a very low density, only detectable by super-resolution $d$ STORM microscopy. These patients might be candidates for therapy with CD19 CART cells. Our data rationalize anti-myeloma responses that have been reported after CD19CART therapy. $d$ STORM analysis allowed defining the threshold of antigen expression for $\mathrm{T}$ cell activation via a CD19CAR, which was found to be less than 100 molecules per cell.

\section{ICLE8-0043}

Hematological malignancies

\section{ENGINEERING CD38 LOW NATURAL KILLER CELLS TO TRANSIENTLY EXPRESS CD16 (F158V) M-RNA ENHANCES THE CYTOTOXIC POTENTIAL OF DARATUMUMAB AGAINST MULTIPLE MYELOMA WITH MINIMAL NK CELL FRATRICIDE}

\author{
S. Sarkar ${ }^{1}$, S. Chauhan ${ }^{1}$, A. Natoni ${ }^{1}$, J. Daly ${ }^{1}$, \\ R. Henderson ${ }^{1}$, M. O'Dwyer ${ }^{1}$ \\ ${ }^{1}$ National University of Ireland-Galway, Apoptosis Research \\ Centre, Galway, Ireland
}

Background: Multiple Myeloma (MM) is a clonal plasma Bcell malignancy and is associated with strong expression of the CD38 antigen. Recent clinical trials with the anti-CD38 monoclonal Antibody (moAb) Daratumumab (Dara) have shown promising results. NK cells are an important immune effector of moAb therapy, mediating ADCC via Fc $\gamma$ RIII (CD16). However, clinical data indicate rapid depletion of CD38 expressing NK cells in patients receiving Dara, questioning the role of $\mathrm{NK}$ cells in disease control. Therefore, we hypothesize that combination of Dara with CD $38^{\text {low }}$ NK cells transiently expressing CD16 m-RNA could offer a novel therapeutic strategy for treatment of MM.

Methods: m-RNA transcripts for CD16 (F158V) protein was synthesized using in-vitro transcription (IVT). CD38 ${ }^{\text {low }}$ KHYG1 NK cells were electroporated with CD16 (F158V) mRNA. KHYG1 cells expressing CD16 (F158V) were subsequently cocultured with MM cells at various E:T ratio in a 14-hour assay.

Results: MM cell lines were classified as CD $38^{\text {hi }}$ (RPMI 8226, H929), and CD38 ${ }^{\mathrm{lo}}$ (JJN3, U266) by flowcytometry. KHYG1 NK cell line had significantly lower CD38 expression as compared to primary expanded NK cells and NK-92 cell line (8-10 fold lower). CD16 ${ }^{+} \mathrm{KHYG1}$ in-combination with Dara were significantly (1.1-4.1 fold) more cytotoxic towards CD $38^{\text {hi }}$ and CD38 ${ }^{\text {lo }}$ MM cell lines as compared to mock transfected KHYG1 with Dara $(n=4)$. Importantly, Dara had no significant effect on viability of CD38 ${ }^{\text {low }} \mathrm{CD} 16^{+}$KHYG1. Additionally, KHYG1 cells maintained their CD16 expression $\left(>85 \%\right.$ CD16 ${ }^{+}$cells) until 120 hours post-transfection $(n=3)$. Furthermore, the combination of
CD16 ${ }^{+}$KHYG1 with Dara was more cytotoxic (57-76\%; newly diagnosed $\mathrm{MM})(36-48 \%$; relapsed $\mathrm{MM})$ to $\mathrm{CD} 38^{+}$primary $\mathrm{MM}$ patient derived cells as compared to mock KHYG1 with Dara (3642\%; newly diagnosed MM) (20-25\%; relapsed MM).

Conclusions: This study provides the proof-of-concept for combining "off-the-shelf" CD38 ${ }^{\text {low }}$ NK cells transiently expressing CD16 (F158V) to enhance the therapeutic activity of Dara in MM patients.

\section{ICLE8-0027}

Hematological malignancies

\section{POST-TRANSPLANT IMMUNOTHERAPY WITH WT1-SPECIFIC CTLS FOR HIGH-RISK ACUTE MYELOGENOUS LEUKEMIA: A PROSPECTIVE CLINICAL PHASE I/II TRIAL}

H.J. Kim ${ }^{1}$, H.J. Sohn ${ }^{2}$, J.A. Hong ${ }^{2}$, H.J. Lee ${ }^{2}$, D.H. Sohn ${ }^{3}$, C.A. Shin ${ }^{3}$, S.J. Hyun ${ }^{3}$, C.H. Hong ${ }^{3}$, H. Choi ${ }^{3}$, A.R. Shin ${ }^{3}$, H.I. $\mathrm{Cho}^{2}$, W.S. Min ${ }^{1}$, T.G. Kim ${ }^{3}$

${ }^{1}$ College of Medicine-The Catholic University of Korea, Leukemia Research Institute-Seoul St. Mary's Hospital, Seoul, Republic of Korea ${ }^{2}$ College of Medicine- The Catholic University of Korea, Catholic Hematopoietic Stem Cell Bank, Seoul, Republic of Korea ${ }^{3}$ College of Medicine- The Catholic University of Korea, Microbiology and Immunology, Seoul, Republic of Korea

In this prospective phase I/II clinical trial of Wilms' tumor antigen 1 (WT1)-specific cytotoxic T cell (WT1-CTL) immunotherapy following allogeneic allogeneic hematopoietic stem cell transplantation (HSCT) in 10 adult patients with high-risk acute myelogenous leukemia (AML), WT1-CTLs, including $\mathrm{CD}^{+}$and $\mathrm{CD}^{+}$cells, were infused four times beginning on day +35 posttransplantation. All patients were engrafted successfully, and five were alive at a median follow-up of 127 months (range, 102-130 months). The 8 -year event-free survival rate was $50 \%$ among all patients and $71.4 \%$ among seven patients with first complete remission pre-HSCT. Three patients were initially resistant to standard induction chemotherapies and died of relapse after transplant. Two patients died without evidence of relapse, one of human cytomegalovirus (HCMV) disease and one of bacterial septicemia. The frequencies of $W T 1$-specific $\mathrm{CD} 8^{+}$and $\mathrm{CD} 4^{+} \mathrm{T}$ cells increased in response to fluctuating $W T 1$ antigen levels.

In this study, we demonstrated that adoptive transfer of WT1CTLs is a feasible therapeutic tool with acceptable safety that induces a long-term clinical response in patients with high-risk AML after allogeneic HSCT.

\section{ICLE8-0029}

Non-viral vectors and transposons

\section{GENERATION OF WILMS' TUMOR 1 TCR- REDIRECTED CD4 AND GAMMA-DELTA T CELLS BY RNA ELECTROPORATION AND CO-TRANSFER OF CD8 MRNA}

D. Campillo $^{1}$, G. Roex ${ }^{1}$, H.H. Van Acker ${ }^{1}$, Z.N. Berneman ${ }^{1,2,3}$, E. Lion $^{1,2}$, V.F.I. Van Tendeloo ${ }^{1}$

${ }^{1}$ University of Antwerp, Vaccine and Infectious Disease Institute, Antwerp, Belgium ${ }^{2}$ Antwerp University Hospital, Center for Cell Therapy and Regenerative Medicine, Edegem, Belgium ${ }^{3}$ Antwerp University Hospital, Division of Hematology, Edegem, Belgium

TCR and CAR engineering of CD8 T cells have played a central role in redirecting the specificity of $\mathrm{T}$ cells towards tumorassociated antigens. However, there is growing interest on the 
role of CD4 and gamma-delta $(\gamma \delta)$ T cells for cancer immunotherapy. In this study, our aim was to determine whether electroporation of mRNA encoding for MHC class I-restricted Wilms' tumor 1 (WT1)-specific TCR could be used to redirect the specificity of CD4 and $\gamma \delta$ T cells and whether co-transfection of $C D 8$ mRNA would improve tumor cell recognition and killing. We transfected non- and pre-activated CD4 T cells according to our in-house developed double sequential electroporation method, first with DsiRNA to suppress de novo expression of endogenous TCR and 24 hours later with $\mathrm{WT}_{37-45}$ TCR mRNA (T37) with or without co-transfection of CD8 mRNA (CD8). Gamma-delta $\mathrm{T}$ cells were expanded using zoledronate and electroporated once with T37 mRNA with or without CD8 mRNA (CD8). After electroporation, we obtained high frequencies of transgenic $\mathrm{TCR}^{+}$cells when T cells were activated (CD4 T cells) or expanded ( $\gamma \delta$ T cells). Percentages of CD8-expressing cells were high in both non- and pre-activated CD4 T cells and expanded $\gamma \delta$ T cells. Subsequently, TCR-redirected T cells were co-cultured with peptide-presenting tumor cells. Antigen-specific recognition of tumor cells was confirmed by expression of activation markers and cytokine and granzyme $\mathrm{B}$ secretion in $\mathrm{T} 37+\mathrm{CD} 8$ transfected pre-activated CD $4 \mathrm{~T}$ cells and $\gamma \delta \mathrm{T}$ cells. In summary, as previously described for CD8 T cells, electroporation is also a valid transfection method to efficiently engineer CD4 and $\gamma \delta$ T cells with a transgenic TCR. CD8 co-expression positively influences $\mathrm{T}$ cell avidity and antigen recognition and suggest a possible redirection of CD4 and $\gamma \delta$ T cells into effectorlike T cells. This research shows the potential of these cell types for adoptive cancer immunotherapy.

\section{ICLE8-0037}

Safety switches and controlled expression

\section{ELIMINATION OF CAR-ENGINEERED LYMPHOCYTES BY AUTOLOGOUS ANTI-CAR T CELLS}

S. Koristka $^{1}$, P. Ziller-Walter ${ }^{2}$, A. Feldmann ${ }^{1}$, C. Arndt ${ }^{1}$, A. Kegler ${ }^{1}$, S. Albert ${ }^{2}$, G. Ehninger ${ }^{3,4}$, M. Bornhäuser ${ }^{3,4}$, M. Bachmann ${ }^{1,2,4}$

${ }^{1}$ Helmholtz-Zentrum Dresden-Rossendorf HZDR, Institute of Radiopharmaceutical Cancer Research, Dresden, Germany ${ }^{2}$ University Cancer Center UCC 'Carl Gustav Carus' Technische Universität Dresden, Tumor Immunology, Dresden, Germany ${ }^{3}$ University Hospital 'Carl Gustav Carus' Technische Universität Dresden, Medical Clinic and Policlinic I, Dresden, Germany ${ }^{4}$ German Cancer Research Center DKFZ and German Cancer Consortium DKTK, partner site Dresden, Heidelberg, Germany

The adoptive transfer of chimeric antigen receptor (CAR) $\mathrm{T}$ cells represents one of the fastest growing areas in cancer immunotherapy. Although gene-modified cells have shown unparalleled antitumor efficiency in patients suffering from hematological malignancies, highly potent CAR T cells can cause severe and partly life-threatening on-target and off-target effects including of cytokine release syndrome and neurological toxicity. Consequently, there is an increasing demand for developing effective strategies to selectively eliminate gene-modified cells in vivo.

One possible approach represents the insertion of a targetable moiety into CAR $\mathrm{T}$ cells. In that regard, we previously characterized a small peptide epitope (E-tag) derived from the human nuclear La protein which we incorporated into the extracellular spacer region of CARs. Based on a monoclonal antiLa antibody recognizing this epitope, we generated a CAR construct for specific binding and depletion of E-tag-labelled CAR T cells.

In flow cytometry-based cytotoxicity assays, $\mathrm{T}$ cells redirected via the novel CAR construct selectively eliminated E-tag-expressing CAR T cells whilst cells lacking this epitope on their surface were not attacked. Interestingly, $T$ cell killing was reciprocal and dependent on an intracellular signaling domain as well as the effector to target cell ratio. Our studies further indicate that $\mathrm{T}$ cells expressing high CAR levels were more efficiently depleted than $\mathrm{T}$ cells with low CAR expression. In addition, $\mathrm{CD}^{+}$and $\mathrm{CD}^{+}$target cells were equally well eliminated by both $\mathrm{CD} 4^{+}$and $\mathrm{CD}^{+}$effector T cells.

Altogether, we here provide an approach for specific and efficient depletion of overactive CAR T cells in case patients experience severe side effects. The E-tag can easily be included into all CARs irrespective of the targeted tumor antigen and represents a promising tool to improve safety of cell-based immunotherapies.

\section{ICLE8-0044}

Safety switches and controlled expression

\section{DEVELOPMENT AND CHARACTERIZATION OF NOVEL ANTI-GD2 TARGET MODULES FOR RETARGETING OF UNIVERSAL CAR T CELLS TOWARD GD2 EXPRESSING TUMORS}

\author{
N. Mitwasi ${ }^{1}$, A. Feldmann ${ }^{1}$, R. Bergmann ${ }^{1}$, N. Berndt ${ }^{1}$, \\ C. Rössig ${ }^{2}$, M. Bachmann ${ }^{1,3,4,5}$ \\ ${ }^{1}$ Helmholtz-Zentrum Dresden-Rossendorf HZDR, Institute of \\ Radiopharmaceutical Cancer Research, Dresden, Germany \\ ${ }^{2}$ Münster University Hospital UKM, Department of Pediatric \\ Hematology and Oncology, Münster, Germany ${ }^{3}$ University \\ Cancer Center UCC 'Carl Gustav Carus' TU Dresden, Tumor \\ Immunology, Dresden, Germany ${ }^{4}$ German Cancer Research \\ Center DKFZ, n, Heidelberg, Germany ${ }^{5}$ German Cancer \\ Consortium DKTK, partner site Dresden, Dresden, Germany
}

Although chimeric antigen receptor (CAR) engineered $\mathrm{T}$ cells demonstrated promising therapeutic effects against cancer, they are still associated with adverse side effects which could be life threatening in some cases. Therefore, in our group we have developed a switchable universal CAR T cell platform "UniCAR", which can be repeatedly switched on and off. This system consists of CAR T cells that cannot bind tumor antigens directly but instead they are redirected with a target module (TM). Such TMs are mainly composed of an epitope on one side, which is recognized by the UniCAR $\mathrm{T}$ cells, and on the other side a tumor antigen-binding domain. Once the TM is eliminated, the UniCAR T cells are no more activated. Disialoganglioside GD2 was shown previously to be a very promising target for several tumors such as neuroblastoma and Ewing's sarcomas. Therefore, anti-GD2 TMs were developed and evaluated regarding their functionality. They were shown to be functional in activating the UniCARs to secrete important pro-inflammatory cytokines and to kill GD2 ${ }^{+}$tumor cells both in vitro and in vivo. To further characterize the anti-GD2 TM with PET imaging, it was labeled with radioactive $\mathrm{Cu}^{64}$. The TM showed a specific enrichment at the site of the GD2 ${ }^{+}$ growing tumor, and it was mainly eliminated through the kidneys within half an hour due to its small size. Such short halflife, provides the UniCAR system with the fast safety switch in case any complications occurred in patients treated with the UniCAR T cells. 


\section{ICLE8-0010}

Solid tumours

\section{ACTIVE MODULATION OF THE TUMOR MICROENVIRONMENT BY ENGINEERED T CELLS}

E. Ceccarello $^{1}$, T. Tabaglio ${ }^{1}$, D.W.K. Boon ${ }^{2}$, W.K.L. Teo ${ }^{1}$, D.Z.M. Tan ${ }^{1}$, J.J.Y. Aw ${ }^{1}$, S.S.F. Koh ${ }^{3}$, A. Pavesi ${ }^{1}$, E. Guccione ${ }^{1}$, A. Bertoletti ${ }^{4}$

${ }^{1}$ Agency for Science- Technology and Research, Institute of Molecular and Cell Biology, Singapore, Singapore ${ }^{2}$ Agency for Science-Technology and Research, Institute of High Performance Computing, Singapore, Singapore ${ }^{3}$ Lion TCR Pte LTD, Lion TCR Pte LTD, Singapore, Singapore ${ }^{4}$ Duke-Nus Medical School, Emerging Infectious Disease, Singapore, Singapore

Adoptive immunotherapy with lymphocytes engineered to express CAR or TCR specific for tumour antigens has shown limited efficacy against solid tumours. The increased PD-L1 expression in the tumour microenvironment (TME) might constitute a major cause as it mediates $\mathrm{T}$ cell suppression by interaction with the PD1 molecule expressed on activated $\mathrm{T}$ cells.

In order to produce $\mathrm{T}$ cells capable of altering such immunosuppressive environment, we utilized a modification strategy consisting of co-transfection of human lymphocytes with Antisense Oligonucleotides (AONs) that modify the splicing of target genes concurrently with mRNA coding for HBV-specific TCRs.

Using AONs targeting Perforin or Interferon- $\gamma$ we demonstrated that it is possible to modify both specificity and function of lymphocytes. We showed that the double-engineered T cells were viable and retained their antiviral function. We then verified the efficiency of AONs in inducing exon skipping in the target mRNAs and we found that isoform switching was detectable as early as 6 hours posttransfection and was lost after 24 hours, concurrent with the loss of the exogenous TCR. We then designed an AON altering the splicing of the PD-1 gene by skipping of the exon coding for the transmembrane domain of the protein. The rationale is to induce a local release of soluble PD-1 to neutralize the PD-L1 present in the TME. After AON transfection, we detected a decrease of the membranebound PD-1 isoform and an increase of the soluble isoform in the supernatant of treated $\mathrm{T}$ cells. Functional assays performed in a 3D model of hepatocellular carcinoma showed that AON-modified TCR-redirected $\mathrm{T}$ cells have a better anti-tumour efficiency.

In conclusion, we developed a method to improve the effectiveness of TCR-redirected T cells in situ, through the conversion of PD-1 into a soluble isoform that could reduce the general immunosuppression in the TME and therefore induce a robust anti-tumour response.

\section{ICLE8-0046}

Solid tumours

TARGETING MULTIPLE TUMORS AND VIRAL INFECTED CELLS USING T-CELLS ENGINEERED TO EXPRESS A NCR-BASED CHIMERIC ANTIGEN RECEPTOR

V. Eisenberg $^{1}$, S. Hoogi ${ }^{1}$, S. Mayer ${ }^{1}$, K. Shamalov ${ }^{1}$, T. Barliya ${ }^{1}$, A. Pogrador $^{2}$, C.J. Cohen ${ }^{1}$

${ }^{1}$ Bar Ilan University, The Mina \& Everard Goodman Faculty of Life Sciences, Ramat Gan, Israel ${ }^{2}$ Ben-Gurion University of Negev, Faculty of Health Sciences, Beer Sheva, Israel

Current developments in cancer treatment are demonstrating the powerful potential of immunotherapeutic strategies. In this regard, autologous transplantation of the T-cells, genetically engineered to express cancer-specific receptors, such as the scFvbased anti-CD19 chimeric antigen receptor (CAR), continues to give a promise for the treatment of haematological malignancies. Still, there is a crucial need to improve the CAR-T cells persistence in patients and to develop efficient CAR-T cell approaches for the treatment of solid tumours.

In parallel, it has been shown that other lymphocytes such as natural killer $(\mathrm{NK})$ cells can demonstrate potent antitumor function. Nonetheless, their use in immunotherapy is rather limited due to difficulties in expanding these cells to therapeutically relevant numbers and to suppression by endogenous inhibitory mechanisms. Cancer recognition by $\mathrm{NK}$ cells is partly mediated by molecules termed natural cytotoxicity receptors (NCRs).

In the present study, we hypothesize that it is possible to endow T-cells with an NK recognition pattern, providing them with a mean to recognize tumour cells in a non-MHC restricted way. To test this, we genetically modified human T-cells with different non-scFv chimeric receptors based on the human NCR2 and NCR1 molecules and then assessed their antitumor activity in-vitro and in-vivo.

Our results show that expression in primary lymphocytes of the non-standard NCR2-derived CAR, termed s4428z and NCR1based CAR, termed h4628z, confers T-cells with the ability to specifically recognize heterogeneous tumours and to mediate tumour regression in a xenograft model.

As viral infected cells can also express NCR ligands, we subsequently tested and demonstrated the ability of NCR-based CAR-T cells to efficiently target flu-infected cells suggesting the feasibility of another possible use of this kind of immunotherapy for fatal human viral infections.

These studies demonstrate the benefit of combining tumour recognition capability of $\mathrm{NK}$ cells with $\mathrm{T}$ cell effectiveness to improve cancer immunotherapy.

\section{ICLE8-0032}

Solid tumours

\section{FINE-TUNING OF CD28 SIGNALING MOTIFS INCREASES ANTI-TUMOR ACTIVITY AND PERSISTENCE OF CHIMERIC ANTIGEN RECEPTOR-MODIFIED T CELLS}

S. Guedan $^{1,2}$, A. Posey ${ }^{2}$, A. Wing ${ }^{2}$, V. Casado-Medrano ${ }^{3}$, C. Shaw ${ }^{2}$, R.M. Young ${ }^{2}$, C.H. June ${ }^{2}$

${ }^{1}$ IDIBAPS, Oncology and Hematology, Barcelona, Spain

${ }^{2}$ University of Pennsylvania, Center for Cellular

Immunotherapies, Philadelphia, USA ${ }^{3}$ University

of Pennsylvania, Department of Systems Pharmacology

and Translational Therapeutics, Philadelphia, USA

The limited expansion and persistence of CAR-T cells in solid tumors is a challenge to the field. We previously showed that the optimization of CAR constructs is essential to increase $\mathrm{T}$ cell persistence. CAR-T cells containing the ICOS and/or 4-1BB intracellular domains have shown improved persistence when compared to CD28 in animal models of cancer. CD28 and ICOS are both members of the CD28 family and they both bind PI3K through their YxxM motif. Here, we hypothesized that replacement of the YMNM CD28 motif for the YMFM ICOS motif within the CAR could improve the persistence of CAR-T cells.

We show that signaling through the mutated CD28-based CAR (28z-YMFM) induced enhanced Akt phosphorylation with 
reduced PLCg and Vav phosphorylation when compared to conventional CD28-based CARs (28z). Despite these differences in T cell signaling, we could not observe any significant differences between the $28 \mathrm{z}$ and the $28 \mathrm{z}$-YMFM CAR-T cells in terms of in vitro cytokine release or killing. We also investigated the therapeutic potential of the CD28 mutant when compared to CD28 and ICOS-based CARs (ICOSz). Treatment with $28 \mathrm{z}$ showed an initial decrease in tumor burden, but $\mathrm{T}$ cells did not persist and tumors eventually progressed. Animals treated with ICOSz showed a slower antitumor effect with enhanced persistence, that was not sufficient to induce complete responses. Interestingly, mutation of the CD28 motif enhanced $\mathrm{T}$ cell persistence, which resulted in complete responses in all the animals treated.

All together, these results indicate that the poor persistence of conventional CD28-costimulated CAR T cells can be enhanced through mutation of the Grb-2 interacting residue, a genetic manipulation that can also increase the anti-tumor activity of the CAR T cells.

\section{ICLE8-0042}

\section{Solid tumours}

\section{CAR-T CELL IMMUNOTHERAPY IN SOLID TUMORS: COLORECTAL CANCER}

E. Ponterio $^{1}$, C. Valvo ${ }^{1}$, M. Cappellari ${ }^{2}$, L. Pasquini ${ }^{2}$, A. Boe ${ }^{2}$, P. Romania ${ }^{2}$, E. Petrucci ${ }^{2}$, C. Amoreo ${ }^{3}$, M.R. Sciuto ${ }^{2}$, R. Dattilo ${ }^{4}$, E. Pilozzi ${ }^{5}$, L. Ricci-Vitiani ${ }^{2}$, M. Biffoni ${ }^{2}$, R. De Maria ${ }^{1}$, T.L. Haas ${ }^{1}$

${ }^{1}$ Catholic University of the Sacred Heart, Institute of General Pathology, Rome, Italy ${ }^{2}$ Istituto Superiore di Sanità, Department of Oncology and Molecular Medicine, Rome, Italy " ${ }^{3}$ "Regina Elena" National Cancer Institute, Department of Pathology, Rome, Italy " Regina Elena" National Cancer Institute, Unit of Tumor Immunology and Immunotherapy, Rome, Italy ${ }^{5}$ Sapienza University, Department of Experimental Medicine, Rome, Italy

At present, FDA-approved chimeric antigen receptor (CAR) T cell-based therapies are limited to hematological malignancies, while patients with solid tumors have not been successfully treated with this approach. One of the main difficulties is the lack of specific targets in these cancer types.

In our study, we aimed to identify novel tumor-specific antigens on the surface of Colorectal Cancer (CRC) stem cells (SCs) to specifically target CAR-T cells. We chose this model, because CRC SCs play a vital role in CRC tumor initiation and progression. We immunized mice with CRC-CRs and screened for hybridomas that produced monoclonal antibodies (mAbs) binding to the surface of primary CRC-SCs but not to peripheral blood leukocytes by high-throughput flow cytometric screening (FACS).

The specificity of mAbs binding was further tested by IHC analyses on frozen and on formalin-fixed paraffin-embedded (FFPE) CRC tissue sections. Finally, we performed surface FACS analyses on freshly dissociated tumors and compared the binding pattern of the mAbs with the binding to normal mucosa of patients. Additionally, we performed FACS analyses with several commercial cell lines and CSCs obtained from different solid tumor types. All these experiments suggested that the selected mAbs selectively bind CRC and, importantly, spare normal colon mucosa.

Using the sequence information of these tumor-specific mAbs, we designed novel CAR constructs targeting the human CRC-CSCs. As a transfer method, we will employ a third-generation lentiviral vector (LV) carrying a synthetic promoter capable of robust and coordinated expression of the CAR molecule (2nd and 3nd generations) and a fluorescent reporter gene to allow the tracking of transgenic $\mathrm{T}$ cells. In the next steps we are going to test these CAR constructs in vitro and in vivo. In conclusion the isolated $\mathrm{mAbs}$ could represent a powerful new tool to redirect $\mathrm{T}$ cells against CRC.

\section{ICLE8-0013}

\section{Solid tumours}

\section{ADOPTIVE T-CELL IMMUNOTHERAPY FOR THE TREATMENT OF GLIOBLASTOMA}

L. Rousso Noori $^{1}$

${ }^{1}$ The George S. Wise Faculty of Life Sciences-Tel Aviv University, Department of Biochemistry and Molecular Biology, Tel Aviv, Israel

Glioblastoma (GBM) is the most common and lethal form of brain cancer. Despite optimal treatment, the median survival of patients diagnosed with GBM is $12-15$ months due to therapeutic resistance and tumor recurrence after surgical removal. Cancer immunotherapy holds a lot of potential as a targeted therapy designed with high affinity to locate specifically the tumor cells and act directly on them, however, its applicability in the GBM context it seems limited, in part, by the lack of ubiquitously expressed tumor antigens. Our aim is to design chimeric-antigen receptor (CAR)engineered $\mathrm{T}$ cells which will be able to recognize novel GBM markers, home to the tumor site and eliminate the tumor cells.

Previously we identified P32/gC1qR/HABP, a mitochondrial protein that is expressed at the cell surface of tumor cells and tumor derived endothelial cells, as a glioma cell surface-marker that could be targeted by immunotherapy. Human and murine glioma cells were evaluated for expression of $\mathrm{p} 32$ by flow cytometry and confocal microscopy. A p32-specific CAR was generated by fusing the scFv to CD28 and FcR-gsignaling domains. Human and murine p32-specific CAR engineered lymphocytes were tested in vitro against human and murine glioma lines (killing assay, IFN-gexpression). The antitumor efficacy of this CAR is currently being examined in a mouse GBM model.

\section{ICLE8-0035}

\section{Solid tumours}

\section{IMPROVING CEA-TARGETED CAR-T CELL THERAPY FOR SOLID TUMOURS}

\author{
W. Zheng ${ }^{1}$, G. Kueberuwa ${ }^{1}$, E. Cheadle ${ }^{1}$, A. Armstrong ${ }^{2}$, \\ R.E. Hawkins ${ }^{1}$ \\ ${ }^{1}$ University of Manchester, Department Cancer Sciences, \\ Manchester, United Kingdom ${ }^{2}$ The Christie NHS Foundation \\ Trust, Department of Medical Oncology, Manchester, United \\ Kingdom
}

Recently, CD19-specific CAR-T cell therapy has shown outstanding results in the treatment of haematological malignancies such as refractory or relapsed B-cell Acute Lymphoblastic Leukaemia (B-ALL). However, the general outcome of CAR-T cell therapy for solid tumours has largely been disappointing to date. Apart from the paucity of tumour-specific target antigens, preclinical studies suggest that this is partly due to several immunosuppressive mechanisms in tumours, which can hinder CAR-T cell homing, intra-tumoural penetration, persistence and effector functions.

The aim of the project is to improve CAR-T cell therapy targeting carcino embryonic antigen (CEA) for solid tumours. To achieve this, my project seeks to establish immunocompetent mouse models to examine the factors that inhibit CAR-T cell 
activity in solid tumours and generate different CARs specific for human CEA with fully mouse intracellular stimulatory domain $\mathrm{CD} 3 \zeta$ in combination with a co-stimulatory domain CD28 or $41 \mathrm{BB}$ to achieve a greater $\mathrm{T}$ cell activation and subsequent anti-tumour activity. To overcome the immunosuppressive tumour microenvironment, immune modulatory CARs which can additionally secrete pro-inflammatory cytokines such as IL-12 or antibodies blocking TGF- $\beta$ or immune checkpoints such as PD-1 were further generated to facilitate CAR-T cell activity.

Current results show that incorporation of the CD28 costimulatory domain improves CAR T-cell anti-tumour activity in vitro. Preliminary data also indicates that the secretion of IL12 from within CEA-specific CAR-T cells enhances their ability to kill tumour cells. In addition, modification to secret an antibody fragment that blocks TGF- $\beta$ or another antibody fragment blocking PD- 1 both seem to confer resistance of CAR T-cells to immune suppression. Importantly, the combination of IL12 cytokine and co-stimulatory domain CD28 achieved promising tumour killing in vivo.

ICLE8-0017

Target and receptor discovery

SURFACE MOBILITY AND CLUSTER FORMATION OF VARIOUS MELANOMA ASSOCIATED ANTIGENS MODULATES CHIMERIC ANTIGEN RECEPTOR (CAR) MODIFIED T CELL ACTIVATION

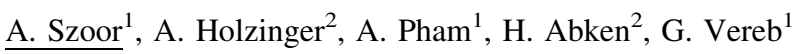

${ }^{1}$ University of Debrecen, Department of Biophysics and Cell Biology, Debrecen, Hungary ${ }^{2}$ University of Cologne Center for Molecular Medicine, Department of Internal Medicine, Cologne, Germany

CAR modified $\mathrm{T}$ cells have been successful for treatment of various B-cell leukemias, however solid cancers failed to respond significantly to CAR T cell therapy. Understanding the molecular mechanisms of action might help us overcome some of the obstacles.

High molecular weight-melanoma-associated antigen (HMWMAA) and melanotransferrin (p97) have similar expression patterns on the tumor cell surface; however, anti-p97 CARs appear to form larger synapses and induce stronger specific cytolytic activation of $\mathrm{T}$ cells than CARs targeting HMW-MAA with similar affinity and specificity. In this study, we have investigated whether different cluster formation of MAAs on the target cell has any effect on synapse formation, or the cytolytic function on the effector $\mathrm{T}$ cell.

Using confocal microscopy and fluorescence correlation spectroscopy on immunostained Melur target cells we found that p97 antigens form smaller clusters (CD: $\sim 280 \mathrm{~nm}$ ) in the membrane and diffuse more rapidly (DT: $44 \pm 22 \mathrm{~ms}$ ) than HMW-MAA molecules (CD: $\sim 740 \mathrm{~nm}$; DT: $124 \pm 42 \mathrm{~ms}$ ). Cross-correlation analysis has shown significant overlap $(\mathrm{C}=0.46 \pm 0.06)$ between HMW-MAA clusters and GM1 positive membrane microdomains (lipid rafts), and less overlap $(\mathrm{C}=0.21 \pm 0.03)$ for $\mathrm{p} 97$. Following 30 minutes $5 \mathrm{mM}$ methyl- $\beta$-cyclodextrin (MBCD) treatment of melanoma cells, GM1 domains were disrupted, which resulted in decreased colocalization of HMW-MAA with lipid rafts $(C=0.24 \pm 0.09)$ and increased diffusional mobility. MBCD treatment of target cells also improved synapse formation and activation of HMW-MAA specific CAR T cells.

In summary, the smaller, more mobile, raft independent p97 antigen clusters induce better CAR T cell activation than the larger, less mobile, raft anchored HMW-MAA. MBCD treatment of target cells disrupts large HMW-MAA clusters, resulting in larger CAR specific immune synapses and more potent $T$ cell activation. Our in vitro findings therefore suggest that a thorough biophysical characterization of the target antigen mobility and association could become an important step in designing CAR $\mathrm{T}$ cell therapies.

\section{ICLE 2018 Standard Poster Presentations}

\section{ICLE8-0014}

Genome editing for allogeneic ACT

TIM-3 TARGETING CAR-T CELLS, AS A POTENTIAL IMMUNOTHERAPY FOR AML

\author{
S. Lee $^{1}$, Z.Y. Ye ${ }^{1}$, H. Huang ${ }^{1}$, M.K. Soh ${ }^{1}$, Cheng-I Wang ${ }^{1}$ \\ ${ }^{1}$ ASTAR, Singapore Immunology Network SIgN, Singapore, \\ Singapore
}

Acute myeloid leukemia (AML) is the most common acute leukemia in adults and among the most lethal. Despite active research in improving outcomes in AML, the standard therapy for AML has not changed much for the past four decades. Developing viable and effective therapeutics for patients with AML has been challanging due to biological heterogeniety and lack of true tumor marker. Allogeneic hematopoietic stem cell transplantation (HSCT) remains the only viable treatment option for AML, and only a limited number of patients qualify. Moreover, $50-70 \%$ of patients relapse after chemotherapy and HSCT, with the 5year survival rate at a dismal $27 \%$. It is known that leukemic stem cells (LSCs), a rare population remains unaffected by chemotherapies, also plays an important role in disease progression and relapse. Hence there remains a clear need to advance treatment strategies and a more individualized approach for the treatment of AML in order to overcome the shortcomings of current therapy.

We aimed to develop adoptive immunotherapy using chimeric antigen receptor (CAR) T-cell therapy technology in attempt to target and kill TIM3-positive AML cells including LSCs. TIM-3 has been previously identified as a surface molecule specific to AML LSCs and its signaling has some function to maintain AML-LSCs. The self-renewing LSCs that survive the intensive chemotherapies may have given rise to recurrence. To eradicate the AML LSC without killing normal HSCs, anti-TIM-3 CAR Tcell therapy can be promising, especially for the patients with relapsed AML. 


\section{ICLE8-0022}

Hematological malignancies

\section{DETECTION NPM1 MUTATION AND CLINICAL CHARACTERS IN ADULT PATIENTS WITH ACUTE MYELOID LEUKEMIA IN THE MONGOLIA}

\author{
$\underline{\text { S. Oyunbileg }}^{1,2}$, B. Davaabayar ${ }^{2}$, N. Oyunderlger ${ }^{1}$, \\ B. Sevjidmaa ${ }^{2}$
}

${ }^{1}$ Center of hematology and bone marrow transplantation, The First central hospital og Mongolia, ulaanbaatar, Mongolia ${ }^{2}$ Physiology, Mongolian National Unniversity of Medical Science, ulaanbaatar, Mongolia

Objective: The NPMc+ is characterized by a wide morphological spectrum of AML subtype, multilineage involvement, absence of CD34, has a distinct gene expression profile, high frequency of FLT3-ITD mutations, and relatively good response to induction therapy, but the favorable overall survival (OS) was found only in the absence of FLT3-ITD. The incidence of NPMI mutations increases $25 \%-35 \%$ of adult AML (45.7\%-63.8\% of adult AML-NK). Our study aims to establish a conventional detection method for NPMI mutation in adult patients with acute myeloid leukemia in the Mongolia.

Materials and methods: Genomic DNA was extracted from peripheral blood of 24 patients newly diagnosed and was diagnosed with acute myeloid leukemia. Primers were designed to distinguish between wild-type NPM1 and NPM1 mutations variants. Methods using a polymerase chain reaction (PCR), PCRABI 3100 Genetic Analyzer (ROX-500 size standard).

Result: NPM1 mutations were found in $8.3 \%$ (2) of patients who have FLT3/ITD was associated with clinical characteristics including higher white blood cell count than acute myeloid leukemia patients without NPM1 mutation. By the KaplanMeyer method, over $40 \%$ of the Adult patients with acute myeloid leukemia overall survival was more than 400 days. In the group of NPM1 mutation with FLT3/ITD overall survival was less than 83 days.

Conclution: The average age of AML morbidity in adult Mongolia is 35 years, and this type of cancer is relatively young. The overall survival rate is over $40 \%$ longer than 400 days, which is longer than the previous years. It is concluded that the treatment of this leukemia is improved. In our study, NPMI was detected $8.3 \%$ patients who had FLT3/ITD mutation.. This type of mutation was low in Mongolia. It is statistically significant to the number of blood cells in diagnosis compared to uneven prevalence groups, the same as in other researchers.

\section{ICLE8-0034}

Delegate Abstract

Hematological malignancies

DETECTION FLT3/ITD MUTATION AND CLINICAL CHARACTERS IN ADULT PATIENTS WITH ACUTE MYELOID LEUKEMIA IN THE MONGOLIA

\author{
S. Oyunbileg $^{1}$, B. Davaabayar ${ }^{2}$, B. Khishigjargal ${ }^{1}$, \\ N. Oyundelger ${ }^{1}$, B. Sevjidmaa ${ }^{2}$ \\ ${ }^{1}$ Center of hematology and bone marrow transplantation, The \\ First central hospital og Mongolia, ulaanbaatar, Mongolia \\ ${ }^{2}$ Physiology, Mongolian National Unniversity of Medical \\ Science, ulaanbaatar, Mongolia
}

Objective: FLT3 is a cytokine receptor that is expressed on leukemic blasts in most cases of acute myeloid leukemia. The
FLT3 gene encodes a tyrosine kinase receptor that plays a key role in controlling survival, proliferation and differentiation of hematopoietic cells. FLT3 mutations have emerged as important targets for molecular therapy.[1] Our study aims to establish a conventional detection method for FLT3/ITD mutation in adult patients with acute myeloid leukemia in the Mongolia.

Materials and methods: Genomic DNA was extracted from peripheral blood of 24 patients newly diagnosed and was diagnosed with acute myeloid leukemia. Primers were designed to distinguish between wild-type FLT3 and FLT3/ITD variants. Methods using a polymerase chain reaction (PCR), PCR-ABI 3100 Genetic Analyzer (ROX-500 size standard), and PCR agarose gel electrophoresis were compared.

Result: FLT3/ITD mutations were found in $12.5 \%$ (3) of patients. FLT3/ITD was associated with clinical characteristics including higher white blood cell count than acute myeloid leukemia patients without FLT3/ITD mutation. By the Kaplan-Meyer method, over $40 \%$ of the Adult patients with acute myeloid leukemia overall survival was more than 400 days. In the group of FLT3/ITD mutation overall survival was less than 100 days.

Conclution: The average age of AML morbidity in adult Mongolia is 35 years, and this type of cancer is relatively young. The overall survival rate is over $40 \%$ longer than 400 days, which is longer than the previous years. It is concluded that the treatment of this leukemia is improved. In our study, FLT3/ITD was detected $12 \%$. This type of mutation was low in Mongolia. It is statistically significant to the number of blood cells in diagnosis compared to uneven prevalence groups, the same as in other researchers.

\section{ICLE8-0018}

Other

\section{CUMULATIVE CHEMOTHERAPY DEPLETES NAIVE T CELLS : IMPLICATIONS FOR CELLULAR THERAPY}

D. Barrett ${ }^{1}$, R. Das ${ }^{1}$, J. Storm ${ }^{1}$

${ }^{1}$ Children's Hospital of Philadelphia, Oncology, Philadelphia, USA

Cellular therapy using engineered $\mathrm{T}$ cells has demonstrated clinical efficacy in the treatment of hematologic malignancies. Recent data suggests the pre-manufacture phenotype may predict ultimate clinical response with standard manufacturing, with Naïve T cells (Singh et al, STM, 2016) and Naïve-like CD27+CD45RO-CD8+ T cells (Fraietta et al, Nat Med, 2018) both implicated.

We collected peripheral blood samples from 157 pediatric patients with acute lymphoblastic leukemia (ALL), non-Hodgkin Lymphomas (NHL), neuroblastoma, osteosarcoma, rhabdomyosarcoma, Wilms, Hodgkins, acute myeloid leukemia (AML) and Ewings sarcoma at diagnosis and after each cycle of chemotherapy. We depleted the adherent cells from this collection, quantified the memory subsets of the CD3+ population using flow cytometry, and expanded these T cells using CD3/CD28 stimulatory beads as in CAR T cell manufacturing. Pre-chemotherapy subset distribution varied with diagnosis, though generally Naïve T cells represented $20-50 \%$ and correlated with potential to expand with CD3/28 beads. The decline in Naïve T cells was consistent across chemotherapy regimens with the exception of standard risk ALL and Wilms tumor who retained high levels of Naïve $T$ cells throughout. Rhabdomysarcoma patients showed a peak/valley response depending on the proximity to cyclophosphamide therapy. Ewings sarcoma therapy was particularly devastating to Naïve T cells, dropping from $28.7 \%$ 
pre-chemotherapy to less than $1 \%$ after 9 cycles with a corresponding complete failure to respond to CD3/28 stimulation. Generally, a rise in Terminal Effector offset the decline in Naïve $\mathrm{T}$ cells, though there was variability between disease types/chemotherapy given.

These data represent the most detailed analysis of $\mathrm{T}$ cell number and phenotype from pediatric cancer patients. The selective effect of depletion of Naïve and Naïve-like $T$ cells from the peripheral blood of pediatric patients may be critical when considering cellular therapies, as more intensive chemotherapy regimens in solid tumors may represent new barriers to efficacy.

\section{ICLE8-0036 \\ Other}

\section{EXPRESSION AND FUNCTIONAL DYNAMICS OF TRPV1 ION CHANNEL IN THE CANINE PBMC}

\author{
J. Bujak $^{1}$, P. Bednarczyk ${ }^{2}$, D. Kosmala ${ }^{1}$, I. Szopa ${ }^{1}$, \\ K. Majchrzak ${ }^{1}$ \\ ${ }^{1}$ Warsaw University of Life Sciences, Department of \\ Physiological Sciences, Warsaw, Poland ${ }^{2}$ Warsaw \\ University of Life Sciences, Department of Biophysics, \\ Warsaw, Poland
}

Ion channels play important role in many biological processes including cell signaling, cellular secretion, proliferation and apoptosis. Transient Receptor Potential (TRP) ion channels are family of six membrane-spanning domain, non-selective cation channels which recently were demonstrated to be expressed in immune cells. However, their exact role in immunity has yet to be determined. TRP ion channels are polymodal cellular sensors activated by temperature, $\mathrm{pH}, \mathrm{ROS}$ and active compounds from plant (e.g. capsaicin, menthol, eugenol). Capsaicin receptor (TRPV1), is the most studied member of the TRP ion channels family. It is speculated that TRPV1 has an effect on NFAT, NF-kB, or Ap-1 pathways, known to be associated with cytokine secretion, cell survival and proliferation via calcium signaling. TRPV1 might tune the immune response and hence, it offers a potential target for immunomodulation.

The main objectives of the study were: 1) to evaluate the mRNA and protein expression of TRPV1 in the canine peripheral blood mononuclear cells (PBMC), 2) to assess whether the expression is affected by temperature or $\mathrm{T}$ cell mitogen treatment and 3) to determine whether TRPV1 activator and blocker influence $\mathrm{T}$ cells activation.

Among vanilloid subfamily of TRP ion channels, TRPV1, -V2, $-\mathrm{V} 3$ and $-\mathrm{V} 4$ transcripts were detected in the canine PBMC. mRNA expression of TRPV1 was not significantly influenced by temperature $\left(33^{\circ} \mathrm{C}, 38.5^{\circ} \mathrm{C}, 41^{\circ} \mathrm{C}\right)$, however mitogen treatment significantly downreguleted expression of TRPV1 on both mRNA and protein level. In silico sequence analysis of canine TRPV1 capsaicin binding site indicated that canine TRPV1 is responsive to capsaicin, a selective TRPV1 activator. Administration of activator (capsaicin) and blocker (capsazepine) led to decrease in T cells activation. Capsaicin treatment also led to downregulation of TRPV1 protein expression, which might partially explain a phenomenon known as desensitization but also drop in $\mathrm{T}$ cell activation.

Research supported by National Science Centre, Poland, 2016/21/N/NZ6/02137

\section{ICLE8-0011 \\ Other}

CLINICAL-GRADE ASPERGILLUS-SPECIFIC T CELLS: SWITCH FROM MAGNETIC SELECTION WITH LYSATE TO SHORT TERM EXPANSION PROCEDURE WITH PEPTIDE POOLS

C. Hoffmann-Freimüller ${ }^{1}$, R. Geyeregger ${ }^{1}$, S. Tischer ${ }^{2,3}$, N. Frank ${ }^{1}$, J. Stemberger ${ }^{1}$, B. Maecker-Kolhoff ${ }^{3,4}$, R. Blasczyk ${ }^{2,3}$, G. Fritsch ${ }^{1}$, B. Eiz-Vesper ${ }^{2,3}$

${ }^{1}$ St. Anna Kinderkrebsforschung e.V.- Children's Cancer Research Institute, Medical University, Vienna, Austria ${ }^{2}$ Institute for Transfusion Medicine, Hannover Medical School, Hannover, Germany ${ }^{3}$ Integrated Research and Treatment Center IFB-Tx, Hannover Medical School, Hannover, Germany ${ }^{4}$ Department of Pediatric Hematology and Oncology, Hannover Medical School, Hannover, Germany

Background: Aspergillus-fumigatus (Asp) infections constitute a major cause of morbidity and mortality in patients after haematopoietic stem cell transplantation. We obtained the manufactering liscence to generate clinical-grade Asp-specific T-cells via the Interferon-gamma (IFN-g) Cytokine-CaptureSystem (CCS) using a GMP-compliant Asp-specific lysate. Due to an unexpected discontinued production of the lysate, we tested the use of Asp-specific peptide-pools for cytokine-secretionassay (CSA) and the already established GMP-conform shortterm-expansion (STE) method.

Methods: Healthy volunteers of the alloCELL registry were screened for the presence of Asp-T-cells via IFN-gElispot. PBMCs from leukapheresis $(n=8)$ were prepared in Hannover for the IFN-g-CSA, therefore, $1 \times 10^{7}$ cells were stimulated for $16 \mathrm{~h}$ with GMP-conform lysate or for $4 \mathrm{~h}$ with peptide-pools followed by magnetic selection of IFN-gproducing T-cells. For the STE in Vienna, $2 \times 10^{7}$ cells were stimulated for 12 days with either the lysate or peptide-pools. The final cell products were characterized for phenotype and function via flow cytometry, IFN-g-EliSpot and IFN-g/ granzyme-B-FluroSpot.

Results: Asp-T-cells were detectable (detection limit: $>2$ spot forming colonies (SFC) $/ 2.5 \times 10^{5} \mathrm{PBMCs}$ ) in $63 \%$ of all volunteers after lysate stimulation but undetectable in $>85 \%$ of healthy donors if peptide-pools were used. The purity of lysate-stimulated IFN-g+ Asp-T-cells was 49\% (range $17.8 \%$ $84.5 \%$ ), while stimulation with the overlapping peptide pools resulted in a non-satisfactory enrichment. In contrast, the STE revealed highly specific Asp-T-cell SFC/ $10^{5}$ cells) for both the lysate $\left(863 \pm 153 / 10^{5}\right.$ cells $)$ and combined peptide-pools $\left(833 \pm 138 / 10^{5}\right.$ cells), respectively. Independent of the stimuli used, predominantly CD4+ T-cells were expanded $(80 \% \pm 3,3$ vs $78 \% \pm 6,3)$ compared to $\mathrm{CD} 8+\mathrm{T}$-cells $(17,1 \% \pm 3,1$ vs $18,4 \% \pm 5,6)$ among CD3. Target cells were highly functional and cytotoxic as determined by secretion of granzyme-B and IFN-g.

Conclusion: Despite the discontinuation of lysate production and consequently enrichment of Asp-T-cells via the IFN-g CCS, clinical-scale production of peptide pool-expanded Asp-T-cells using the STE method might enable an efficient adoptive T-cell immunotherapy. 


\section{ICLE8-0008}

Other

\section{COMPREHENSIVE ANALYSIS OF STARTING MATERIALS FOR GENE-MODIFIED CELL-BASED IMMUNOTHERAPIES}

\author{
A. Luostarinen ${ }^{1}$, S. Ylä-Herttuala ${ }^{2}$, J. Nystedt $^{1}$, T. Kaartinen $^{1}$ \\ ${ }^{1}$ Finnish Red Cross Blood Service, Advanced Cell Therapy \\ Centre, Helsinki, Finland ${ }^{2}$ A.I. Virtanen Institute for Molecular \\ Sciences-University of Eastern Finland, Department of \\ Biotechnology and Molecular Medicine, Kuopio, Finland
}

For cell-based immunotherapies, blood is the self-evident source of starting material. Although autologous leukapheresis product is the most commonly used starting material, other types of blood products could be preferred depending on the type and clinical status of the donor (autologous/allogeneic, pediatric), as well as blood procurement procedures and equipment available.

Several aspects concerning blood-derived starting materials may affect the manufacturing success of cellular therapeutics: the quality and frequency of the target cell subsets could be affected by the procurement procedure or by early processing steps such as cryopreservation. Furthermore, non-target cells may affect the efficiency of transduction or expansion of the desired cells.

We compared leukapheresis products, buffy coats, and small peripheral blood samples from healthy donors in respect of their usefulness as starting materials for immune cell therapy. The frequency of different leukocyte types and other blood cells were analyzed with Sysmex cell counting before and after mononuclear cell (MNC) isolation or cryopreservation. Further phenotyping of lymphocyte subtypes and monocytes was performed with flow cytometry. Activation and memory status of $\mathrm{T}$ cells was also assessed.

To study the functional effects of different starting materials, we used CAR T cell production as an example for gene-modified immune cell therapy. Cells in the MNC fraction were lentivirally transduced with CD19/CD28/z-CARs and expanded using CD3/ CD28 microbeads and either $20 \mathrm{IU} / \mathrm{ml} \mathrm{IL-2}$ or $10 \mathrm{ng} / \mathrm{ml} \mathrm{IL-7} \mathrm{and}$ IL-15. Cell viability and expansion efficiency were monitored throughout the expansion. $\mathrm{T}$ cells were characterized and their CAR expression measured at day 10 along with in vitro CAR $\mathrm{T}$ cell potency.

This comprehensive analysis of blood-derived starting materials provides new perspectives for the generation of immune cell therapy products. The starting cell material is one of the key, but often overlooked, factor for successful manufacturing of cell-based products.

\section{ICLE8-0020}

Other

\section{OPTIMISATION OF CYTOKINE-DEPENDENT EXPANSION FOR TCR-TRANSDUCED AUTOLOGOUS T-CELLS}

$\underline{\text { R. Petrovic }}^{1}$, D. Biziato ${ }^{1}$, A. Toufexi ${ }^{1}$, J. Kerby $^{1}$

${ }^{1}$ Catapult Cell \& Gene Therapy, Process Development, London, United Kingdom

Cytokines have important functions related to T-cell expansion, differentiation, survival, and homeostasis. IL-2 is the most frequently used cytokine in generating lymphocytes for adoptive immunotherapy, however IL-7, IL-15 and IL-21 have been indicated to promote higher proliferation of CD8+ T cells, as well as their effector functions (i.e., in-vivo persistence, cytokines secretion and cytotoxic activity). A first set of experiments performed using a Design of experiment (DOE) modelling - outlining a full two-level factorial design to investigate the combinatorial desirability of different concentrations of cytokines (IL-2, IL-7, IL-15 and IL-21) highlighted a possible key role of IL-21 in determining yields of transduced CD8+ cells and identified cytokines combinations that would potentially increase proliferation and transduction of CD8+ cells. A follow up experiment was carried out to validate the suitability of Design-Experiment model generated from the analysis to confirm the effect of IL-21 compared to IL-2 on the following T-cell culture parameters; cell viability and expansion fold; transduction efficiency and phenotype, this experiment was carried out using multiple donors and highlighted the impact of donor to donor variability on outcomes. A largescale experiment was also carried out as a direct comparison of a clinical trial process comparing the use of IL-2 and IL-21 and showed that with long term culture IL-21 impacted on cell viability but did not result in any phenotypic differences or benefits. We concluded the high level of variability and additional costs outweighed any potential benefits associated with deviating from the standard protocol of culturing T-cells for autologous therapy in IL-2.

\section{ICLE8-0033 \\ Other}

IMPACT OF THE SIGNAL STRENGTH AND TEMPERATURE DURING T LYMPHOCYTES ACTIVATION AND PROLIFERATION - STUDY ON COMPANION DOG MODEL

\author{
I. Szopa $^{1}$, J. Bujak ${ }^{1}$, A. Łabędź ${ }^{1}$, K. Majchrzak ${ }^{1}$ \\ ${ }^{1}$ Warsaw University of Life Sciences-Faculty of Veterinary \\ Medicine, Department of Physiological Sciences, Warsaw, \\ Poland
}

Companion dog is an attractive model for immunological studies. Major subsets of the dog immune system were characterized with significant homology to humans. Importantly, culture of large amounts of canine $\mathrm{T}$ lymphocytes for the purpose of adoptive cellular immunotherapy still requires optimization. In order to obtain logarithmic expansion of $\mathrm{T}$ cells, a proper activation (engages $\mathrm{T}$ cell receptor - TCR) and costimulation has to be applied. In our study we used nano-sized magnetic beads coated with anti-canine CD3 antibody to trigger the signal mediated by TCR. Moreover, we coated beads with anti-canine CD28, ICOS or 41BB antibody to provide costimulatory signal ensuring immune cells proliferation and cytokine production. We used beads at different concentration: 1:2, 1:1, 1:0.5, 1:0.25 and 1:0.125 $\mathrm{T}$ cell: bead ratio. We evaluated $\mathrm{T}$ cells activation status based on phenotypic features of the T cells and expression of CD25 molecule. Our research shown that low-strength activation signal (1:0.5 ratio) caused increased expression of CD25 molecule on canine $\mathrm{CD}^{+}$and $\mathrm{CD}^{+} \mathrm{T}$ cells surface, 24 and $72 \mathrm{~h}$ post-stimulation. Lower beads concentration made $\mathrm{T}$ lymphocytes to create multiple aggregates, which indicate $\mathrm{T}$ cells activation. Furthermore, activated $\mathrm{T}$ cells produce significant amount of IFN- $\gamma$. The impact of temperature (ranging between $33^{\circ} \mathrm{C}$ to $41^{\circ} \mathrm{C}$ ) on canine $\mathrm{T}$ cells activation was also determined. We found $38,5^{\circ} \mathrm{C}$ as a proper 
temperature for canine $\mathrm{T}$ cell proliferation and functionality. Overall our research revealed the optimal conditions for canine $\mathrm{T}$ cells activation and expansion for further immunological assessment and most importantly for adoptive $\mathrm{T}$ cell transfer therapy purpose. Especially seeing that this treatment gives hope to cure cancer in humans, as well as, in canine patients. The project is carried out within the First TEAM programme of the Foundation for Polish Science co-financed by the European Union under the European Regional Development Fund.

\section{ICLE8-0019}

Solid tumours

\section{GENETIC ENGINEERING OF TUMOUR INFILTRATING LYMPHOCYTES (TIL) WITH A NOVEL GROWTH FACTOR RECEPTOR FOR TREATMENT OF SOLID TUMOURS}

\author{
M. Le Brocq ${ }^{1}$, G. Owens ${ }^{2}$, J. McCaffrey ${ }^{1}$, A. Patel ${ }^{1}$, \\ R.D. Guest $^{1}$, R.E. Hawkins ${ }^{1,2}$, J.S. Bridgeman ${ }^{1}$ \\ ${ }^{1}$ Immetacyte, Research \& Development, Manchester, United \\ Kingdom ${ }^{2}$ University of Manchester, Division of Cancer \\ Sciences, Manchester, United Kingdom
}

Whilst great success has been achieved in treating leukaemia with genetically engineered Chimeric-Antigen-Receptor (CAR) T-cells, there has been limited success in treatment of solid tumours with such single-antigen-specific therapies. Currently, the most successful cell therapy regimens for some solid cancers such as melanoma, involve treatment using Tumour Infiltrating Lymphocytes (TIL), the naturally-occurring polyclonal T-cell population found within the tumour itself. Whilst success rates of TIL treatment in certain tumours are equivalent to those found with other recently-licensed immunotherapies (such as the checkpoint blockade inhibitors), there are still some challenges in making these therapies of benefit in a greater number of tumour types and therefore patients. Some of these are - difficulty in transduction of TIL using specific lentiviral engineering approaches, persistence of TIL after infusion and consistency in initiation of an effective immune response against the tumour. In this work, we present a method of genetic modification of TIL with limited effect on viability, which fits into current GMP protocols for TIL manufacture. We also show how we have used this protocol to test a novel drug-controlled receptor with the potential to work as a costimulatory growth factor receptor in TIL therapies for various solid tumour types.

\section{ICLE8-0009}

\section{Solid tumours}

\section{A CAR-T CELL THERAPY TARGETING GLYPICAN-3 POSITIVE HEPATOCELLULAR CARCINOMA}

B. Wang ${ }^{1}$, E. Ngoh ${ }^{1}$, M.K. Soh ${ }^{1}$, Y. Yeap ${ }^{1}$, H. Huang ${ }^{1}$, H.C. $\operatorname{Tan}^{1}$, Y. $\mathrm{Hu}^{1}$, C.I. Wang ${ }^{1}$

${ }^{1}$ Singapore Immunology Network-Agency for ScienceTechnology and Research ASTAR, Human Monoclonal Antibodies Platform, Singapore, Singapore

Hepatocellular carcinoma (HCC) is the third leading cause of cancer-related death worldwide. The majority of HCC patients are ineligible for curative therapies upon diagnosis, while the systemic therapy or targeted therapy only showed marginal clinical benefit.
Recently, immunotherapies using $\mathrm{T}$ cells expressing chimeric antigen receptor (CAR) have been demonstrated as a promising cancer treatment strategy. Glypican-3 (GPC-3) is a tumour specific antigen that is often overexpressed in $\mathrm{HCC}$ but not detectable in normal adult tissues. Several CAR-T cell therapies targeting GPC3 positive hepatocellular carcinoma are currently in various stages of clinical development. Using phage display and antibody engineering technology, we have constructed a number of antibody clones against human GPC-3 protein, most of which showed strong and highly specific binding to GPC-3 positive HCC cells as well as cross-reactivity with both cynomolgus and mouse GPC-3. A list of clones has been selected to be developed into the second generation CAR-T cells targeting GPC-3 and these CAR-T cells exhibited strong target killing only on GPC-3 positive but not GPC-3 negative cells. Furthermore, when co-cultured with various GPC-3 expressing cells, T cell cytokines such as IL-2 and Interferon- $\gamma$ were detected from the culture supernatant and $\mathrm{T}$ cell blasting was also observed. In future, CAR clones that showed best killing potency and target specificity in vitro will be selected for in vivo mouse experiment for both efficacy and safety issues, and the antiGPC-3 CAR-T cells will also be tested in combination with other therapeutic strategies such as immune checkpoint blockade.

\section{ICLE8-0012}

Target and receptor discovery

\section{OPTIMISING CHIMERIC ANTIGEN RECEPTOR T-CELL THERAPY FOR HAEMATOLOGICAL MALIGNANCIES}

A. Bahashwan $^{1}$, M. Kalaitsidou ${ }^{1}$, A. Schutt $^{1}$, D. Gilham ${ }^{1}$, R. Hawkins ${ }^{1}$

${ }^{1}$ The University of Manchester, Division of Cancer SciencesFaculty of Biology-Medicine and Health, Manchester, United Kingdom

Cancer immunotherapy has been an attractive approach over the last few years as a potential way of treating cancers. One branch is the adoptive T-cell therapy, where T-cells bearing specific anti-tumour activity are infused into patients carrying the tumour. These cells can be isolated from the tumour and called tumour infiltrating lymphocytes, or can be redirected by expressing artificial receptors and referred to as chimeric antigen receptor (CAR) T-cells. CAR T-cells have been studied on haematological malignancies, particularly those expressing CD19 antigen, which led to the FDA approval the 'KYMRIAH' to groups of acute lymphoblastic leukaemia patients. However, $\mathrm{T}$-cell requires a co-stimulation and many receptors are present but the most prominent ones are CD28 and 4-1BB. These receptors have been studied in the context of CD19 CAR T-cells in vitro and in vivo. However, the cytotoxicity and persistence of the infused cells, which are the modulators of an effective CAR T-cell therapy, are still debatable. This study demonstrates a panel of 12 CD19 CARs that have different co-stimulatory domains looking for the differences in response that might suggest an ideal CAR construct. It is also important to define a target that has no/low expression on normal tissue to avoid toxicity, particularly for tumours of myeloid origin. This study describes the oncofoetal antigen, 5T4 as a potential target for acute myeloid leukaemia (AML). 5T4 antigen has a restricted distribution on normal tissues, but is overexpressed in many malignancies. To investigate this antigen, the CAR panel has been sub-cloned into 5T4 looking for the response against AML cells. 


\section{ICLE 2018 Late Breaking Abstracts}

\section{ICLE8-0056}

Hematological malignancies

\section{KINETICS OF EXPANSION AND PHENOTYPE OF UCART19 CELLS IN THE FIRST CLINICAL TRIAL OF A UNIVERSAL ALLOGENEIC CAR-T CELL PRODUCT}

A. Jozwik ${ }^{1}$, C. Graham ${ }^{1}$, A. Dunlop ${ }^{2}$, K. Sanchez ${ }^{2}$, D. Yallop ${ }^{2}$, P. Patten ${ }^{2}$, A. Pagliuca ${ }^{2}$, G. Mufti ${ }^{2}$, L. Gauthier ${ }^{3}$, A. Zinai ${ }^{3}$, S. Dupouy ${ }^{3}$, S. Balandraud ${ }^{3}$, C. Poirot ${ }^{3}$, A. Philippe ${ }^{3}$, F. Simon ${ }^{3}$, C. Konto ${ }^{4}$, B. Ganguly ${ }^{4}$, T. Pertel ${ }^{4}$, B. Sasu ${ }^{4}$, R. Benjamin ${ }^{2}$

${ }^{1}$ King's College London, Department of Haemato-oncology, London, United Kingdom ${ }^{2}$ King's College Hospital NHS Foundation Trust, Haematology, London, United Kingdom ${ }^{3}$ Institut de Recherches Internationales Servier, Translational and Clinical Research, Suresnes, France ${ }^{4}$ Allogene Therapeutics- Inc., Translational and Clinical Research, San Francisco, USA

UCART19, the first off-the-shelf allogeneic CAR-T cell product, is currently in clinical trials involving adult and pediatric patients with relapsed/refractory B-acute lymphoblastic leukaemia. This product, derived from normal donor T-cells, expresses an anti-CD19 scFv with 4-1BB and CD3zeta costimulatory and activation domains respectively and has additional genetic modifications including knockout of TRAC and CD52 genes and a CD20 epitope safety switch. UCART19 expansion, kinetics and immunophenotype were assessed in blood and bone marrow in a cohort of six patients treated at the first dose level $\left(6 \times 10^{6}\right.$ UCART19 cells) following lymphodepletion with fludarabine, cyclophosphamide and an anti-CD52 monoclonal antibody in the dose escalation of the CALM study.

Four out of five evaluable patients at D28 achieved MRD negative $C R$ with manageable safety profile. A single patient was not evaluable at day 28 due to experiencing a dose-limiting toxicity of Grade 4, Cytokine Release Syndrome and Grade 5 neutropenic sepsis at D15. Successful expansion of UCART19 cells was observed in 5 out of 6 patients by flow cytometry and qPCR. Median peak level of $174 \mathrm{CAR}+$ cells/ul was achieved between days 14 and 17, with a decline in numbers to a median of $1.75 \mathrm{CAR}+$ cells/ul by day 28 . In both peripheral blood and bone marrow, CD8 cytotoxic cells were the main population of expanding CAR-T cells, followed by a double negative CD4CD8- population, with only a very low number of CD4+ cells. No expansion of $\mathrm{TCR} \alpha \beta$ CAR+ cells was reported.

Overall our data shows successful expansion of UCART19 even at the lowest dose level in this phase 1 study, with CD8+ T cells as the main population of expanding cells in peripheral blood and bone marrow.

\section{ICLE8-0054 \\ Hematological malignancies \\ CLINICAL MANUFACTURING OF CHIMERIC ANTIGEN RECEPTOR (CAR) T CELLS FOR ADOPTIVE IMMUNOTHERAPY}

\author{
K. Mølgaard ${ }^{1}$, Ö. Met $^{2}$, I.M. Svane ${ }^{1}$
}

${ }^{1}$ Center for Cancer Immune Therapy, Department of Hematology-Herlev Hospital, Herlev, Denmark ${ }^{2}$ Center for Cancer Immune Therapy, Department of Oncology-Herlev Hospital, Herlev, Denmark

Introduction: In Europe, cancer is the leading cause of disease related deaths in children. For high risk leukemia patients that develop primary drug resistance and relapse after receiving allogeneic stem cell transplantation, the overall survival rate is less than $25 \%$. Advances in immunotherapy, like chimeric antigen receptors (CARs), has improved treatment options especially for patients with hematological malignancies. Considering this, we aim to establish facilities for the generation and characterization of CAR T cell for therapy, initially, in children with high risk acute lymphoblastic leukemia using the well documented CD19 targeting CARs (CAR19) as a foundation.

Material and methods: The purpose of this study is to establish in-house facilities for large-scale manufacturing of clinical doses of CAR T cells in minimum time. Furthermore, we aim to optimize in vivo persistence by addressing the metabolic and phenotype characteristics of effective CAR T cell responses.

Results and conclusion: We have shown that the production of CD19 specific CAR T cells is feasible using lentiviral vectors, that $\mathrm{T}$ cell product can be rapidly expanded, and that CD19 CARs kill cancer cells in an antigen-specific manner. Furthermore, analysis of cytokine secretion shows that there is a distinct profile from activated CD4+ and CD8+ T cells with a dominant expression of TNF from CD4+ cells and a mixed co-expression of TNF, IFN $\gamma$ and CD107a from CD8+ cells. Upon stimulation more than $70 \%$ of activated CD4+ cells express TNF, around 30\% of CD8+ cells coexpressed all three activation markers and $30 \%$ expressed TNF plus CD107a.

In conclusion we have shown that we can generate functional CAR T cells which can specifically kill cancer cells upon recognition of the cognate antigen. Preliminary results show a distinct phenotypic profile from CD4+ and CD8+ CAR T cells which could inflict metabolic characteristics and could influence the final CAR $\mathrm{T}$ cell response. 
ICLE8-0055

Other

ROLE PLAY BY GALECTIN-1 IN REGULATORY T-CELL IMMUNOTHERAPY FOR MODULATION OF IMMUNE RESPONSES IN THE TREATMENT OF AUTOIMMUNE DISEASES

R. Fernadez-Perez ${ }^{1,2}$, O. Aberquilla-Fernandez ${ }^{1,2}$, R. Sanchez-Dominguez ${ }^{2}$, J.A. Bueren ${ }^{1,2}$, M.I. Garin ${ }^{1,2}$

${ }^{1}$ CIEMAT/IIS Fundación Jiménez Díaz, Advanced Therapy Unit, Madrid, Spain ${ }^{2}$ Centro de Investigaciones Energéticas Medioambientales y Tecnológicas and Centro de Investigación Biomédica en Red de Enfermedades Raras CIEMAT/CIBERER, Division of Hematopoietic Innovative Therapies, Madrid, Spain

Foxp3-expressing regulatory $\mathrm{T}$ cells (Tregs) are vital for maintaining balance among tolerance, adequate immune responses, and autoimmunity. In recent years, regulatory $\mathrm{T}$ cells are being proposed in regulatory $\mathrm{T}$-cell immunotherapy with the potential to modulated unwanted immune responses. While their homeostasis has been extensively studied, their mechanisms of immunoregulation still remain to be fully clarified. We previously found that galectin-1 (GAL1), a $\beta$-galactoside-binding lectin, is expressed by human and mouse regulatory $\mathrm{T}$ cells and contributes to their immunosuppressive function in vitro. In this study, we examined the prophylactic and therapeutic effects of GAL1-deficient $\mathrm{CD} 4{ }^{+} \mathrm{CD} 25^{+} \mathrm{Foxp}^{+}$regulatory $\mathrm{T}$ cells (GAL1 KO-Tregs) in a T cell transfer in vivo model of chronic inflammation. Our findings demonstrate that GAL1 KO-Tregs retained their capacity to modulate intestinal inflammation induced by FOXP3- ${ }^{-} \mathrm{CD}_{4} 5 \mathrm{Rb}^{+} \mathrm{CD}^{+}$naïve $\mathrm{T}$ cells into Rag-1 mice. However, the immunomodulation achieved was less efficient with respect to wild-type Tregs, as shown by the increase number of GAL1 KO-Tregs required for effective protection against intestinal inflammation. In addition to this, the long term survival of the mice treated with GAL1 KOTregs was compromised when compared to those mice treated with wild-type Tregs. We conclude that galectin-1 plays a critical role in the $\mathrm{T}$ cell mediated regulation of chronic colitis and that an adequate expression of galectin-1 should be taken into consideration in regulatory $\mathrm{T}$-cell immunotherapy for modulation of immune-mediated diseases.

\section{ICLE8-0051}

\section{Other}

\section{CTSTM DYNABEADSTM CD3/CD28 ENABLES SIMULTANEOUS ONE-STEP NAÏVE AND EARLY MEMORY T ISOLATION AND ACTIVATION}

\author{
T.E. Stav-Noraas ${ }^{1}$, R. Hartberg ${ }^{1}$, T. Looney ${ }^{2}$, L. Miller ${ }^{2}$, \\ G. Lowman ${ }^{2}$, H. Almåsbak ${ }^{1}$ \\ ${ }^{1}$ Thermo Fisher Scientific, Cellular medicine, Oslo, Norway \\ ${ }^{2}$ Thermo Fisher Scientific, Clinical Next Generation Sequencing, \\ Carlsbad, USA
}

Following demonstration of the tremendous potency of CAR T-cell therapies in blood cancers, the therapy field is evolving rapidly with focus on commercial, cost-effective T-cell manufacturing processes to offer therapies for larger patient groups. There is a need for shorter and better manufacturing processes, with closed-system operations and simplified workflows. CTSTM DynabeadsTM CD3/CD28 has been used successfully in advancing $\mathrm{T}$ cell therapies through $>88$ clinical trials and lately also in commercial $\mathrm{T}$ cell drug manufacturing.
CTSTM DynabeadsTM CD3/CD28 requires no upstream T cell selection as this technology simultaneously isolates and activates naïve and early memory $\mathrm{T}$ cells based on CD3 and CD28 co-expression. T cells are isolated with high recovery $(>90 \%)$ and purity ( $>95 \%)$ and uniformly stimulated $(>95 \%$ CD25+day 3 post-activation). Activated $\mathrm{T}$ cells expanded in CTSTM OpTmizerTM T Cell Expansion Serum Free Media with 5\% CTSTM Immune Cell Serum Replacement preserve a young phenotype (CD28+CD62L+) at day 7-10 and demonstrate increased clonal diversity compared to the starting material (AmpliSeqTM TCR $\beta$ sequencing). Finally, we show that largescale, closed-system early removal of the beads (days 2, 3, 5 postactivation) is feasible but comes with some cell loss days 2 and 3 (T cell recovery $60-80 \%$ ). In contrast, nearly $100 \%$ of the cells are recovered following bead removal day 5. Surprisingly, a shorter activation period before transduction day 3 post-activation increases $\gamma$-retrovirus transduction efficiency 2 folds. For Research Use or Non-Commercial Manufacturing of Cell-Based Products for Clinical Research. Caution: Not intended for direct administration into humans or animals.

\section{ICLE8-0053}

Safety switches and controlled expression

\section{PRECLINICAL OPTIMIZATION OF CHIMERIC ANTIGEN RECEPTOR CD123-EXPESSING T-CELLS FOR ACUTE MYELOID LEUKEMIA AND INCLUSION OF AN INDUCIBLE CASPASE 9 SUICIDE GENE TO INCREASE SAFETY}

M.L. Baroni ${ }^{1}$

${ }^{1}$ Carreras Leukemia Research Institute, Medicine, Barcelona, Spain

The lack of Acute Myeloid Leukemia (AML)-specific surface markers has delayed the development of Chimeric Antigen Receptor (CAR)-based adoptive immunotherapies for AML. T-cells expressing a CAR specific for AML cells are an attractive targeted-therapy for the treatment of relapsed/refractory AML as well as for myeloablative conditioning regimen prior to hematopoietic stem cell (HSCs) transplantation.

A second generation CARCD123 was generated and cloned in a pCCL lentivector with or without the Inducible Caspase 9 (iC9). A large battery of in vitro and in vivo assays were performed, and are ongoing, to characterize the efficacy and safety of our CARCD123.

Here, we show that CD123 is the most common surface marker in AML and that normal CD34 ${ }^{+}$Hematopoietic Stem Cells (HSCs) express 3-fold lower levels of CD123.

Monocyte-depleted Peripheral Blood Mononuclear Cell (PBMCs) were activated and infected with CARCD123expressing lentiviral supernatants, and $24 \mathrm{~h}$ later CAR transduction was successfully detected in activated $\mathrm{CD} 4^{+}$and $\mathrm{CD} 8^{+}$ T-cells. CARCD123-expressing activated $\left(\mathrm{CD} 69^{+} \mathrm{CD} 25^{+}\right) \mathrm{T}$ cells expanded extensively in vitro, and exerted robust and specific in vitro cytotoxicity over $\mathrm{CD} 123^{+} \mathrm{AML}$ cell lines and primary patient samples associated to a massive release of proinflammatory cytokines (TNF $\alpha$, INF $\gamma$ and IL2). Importantly, CARCD123 T-cells largely targeted normal HSCs as shown by a significant reduction in their clonogeneicity potential as revealed by preliminary CFU assays. The in vivo efficiency and toxicity of CAR123 T-cells is now being tested in NSG mice using cell lines, primary AML samples and Cord Blood-derived CD34 ${ }^{+}$cells. Our current data suggests CAR123 toxicity on CD123 ${ }^{+}$AML cell lines as well as on normal CD34+ HSCs. Thus, in order to circumvent 
the "on-target off-tumor" CARCD123 toxicity on normal HSCs, current efforts in our institute are focused on the inclusion of a safety switch into the lentivector encoding the CARCD123, allowing an on/off control of CAR-expressing T-cells.

\section{ICLE8-0048}

Solid tumours

\section{TUMOR-ASSOCIATED FIBROBLASTS: GENERIC TARGET OF CANCER IMMUNOTHERAPY}

\author{
$\underline{\text { F. Bojin }}^{1}$, O. Gavriliuc ${ }^{1}$, V. Paunescu ${ }^{1}$ \\ ${ }^{1}$ Victor Babes University of Medicine and Pharmacy Timisoara, \\ Immunology, Timisoara, Romania
}

Tumor cell heterogeneity depends on the tissue from where the tumor has originated, the patient's genetic background, the antigenic drift during tumor progression, making them elusive targets for cancer therapy; however, tumor-associated fibroblasts (TAFs) can be conveniently similar in their origin, phenotype and genotype, regardless of cancer type.

The approach focused ondirect detection of endogenous epitope-specific $\mathrm{T}$ cells using methods that distinguishes TCR specificity by its binding to cognate peptide - MHC complexes. CTLs expressing a particular TCR are able to recognize not only tumor cells antigen, but also microenvironment specific antigens, such as those on TAFs.

We used surgical removed fragments of breast cancer to obtain TAFs, which were further cultivated and expanded in vitro in standard culture conditions. From the same patient we obtained peripheral blood mononuclear cells (PBMC) by density gradient centrifugation. On the surface of TAFs we identified by flowcytometry presence of specific antigen - fibroblast activation protein (FAP) and we custom-made MHC I-Strep HLA-A*0201 FAP for isolation of CTLs. Antigen-specific T cell sorting was performed by magnetic nanobeads separation, biotin-binding for removal of magnetic particles, and we performed co-culture of TAFs with positive and negative $\mathrm{T}$ cell fractions. After in vitro expansion of CTLs for 2 weeks, we were able to isolate using Streptamer technology a proportion of $20 \%$ of CD8+ T cells, specific for our antigen. Co-culture of cytotoxic $\mathrm{T}$ lymphocytes was performed in a ratio of 1:1 with TAFs. TAFs proliferation in vitro was abolished after 3 days of co-culture with FAP specific CTLs, compared with non-specific $\mathrm{T}$ cells, which induced only decrease in TAFs proliferation after 5 days of co-culture.

As our on-going research on TAFs uncovers novel antigenic targets, the flexibility of the Streptamer technology will allow us to select even more specific CTLs.

\section{ICLE8-0057}

\section{Solid tumours}

\section{IMPROVING THE EXPANSION AND PERSISTENCE OF CAR T CELLS USING ONCOLYTIC RHABDOVIRAL VACCINE VECTORS IN PAEDIATRIC SARCOMAS}

\author{
$\underline{\text { F. Zemp }}{ }^{1}$, J. Rajwani ${ }^{1}$, K. Potts ${ }^{1}$, S. Van ${ }^{1}$, D. Mahoney ${ }^{1}$ \\ ${ }^{1}$ Alberta Children's Hospital Research Institute- Univeristy \\ of Caglary, Microbiology- Immunology \& Infectious Diseases \\ MIID, Calgary, Alberta, Canada
}

Current treatment methods for paediatric solid tumours suffer from two main deficiencies: they are ineffective against metastatic disease, and chemo/radiotherapy results in long-term morbidities in surviving children. Further, in some paediatric solid tumours (i.e., metastatic sarcomas) survival outcomes have changed little in over three decades.

Alternatively, paediatric blood cancers have been revolutionized through CAR T therapy, but results in solid tumours have been underwhelming. We hypothesize that enhancing CAR $T$ responses in solid tumours with adjuvant oncolytic rhabdoviral vaccine vectors (ORVVs) will expand the CAR $\mathrm{T}$ product in vivo, while conditioning the tumour microenvironment to be more permissive to CAR T therapy.

Using mouse model systems, we have found that adjuvant administration of ORVVs harbouring cognate antigen to the native $\mathrm{T}$ cell receptor (TCR) of the CAR $\mathrm{T}$ cell results in a substantial increase in the CAR T product without compromising the function of the CAR T cell. Subsequent reboosting of the CAR T product with antigenically distinct ORVVs results in further expansion and robust persistence, even in the absence of chemoablation. Using a combination of cell sorting, whole body imaging and intravital microscopy, we have determined that the quality of the CAR T boost is dependent on the ability of the adoptively transferred $\mathrm{T}$ cells to traffic to splenic follicles and access virally infected, antigen-presenting cells. Modulating the ex vivo expansion protocol to enrich memory $T$ cell populations that can traffic to these follicles dramatically improves boosting and reboosting capabilities as well as CAR T persistence.

We have established xenograft and PDX mouse tumour models with haplotype-matched TCRs, and are currently testing the efficacy of this ORVV boosting strategy in these preclinical models.

\section{ICLE8-0050}

\section{Target and receptor discovery}

\section{CELL THERAPY WITH ENGINEERED HLA-A2 SPECIFIC CAR-CD8+TREGS TO AVOID TRANSPLANT REJECTION}

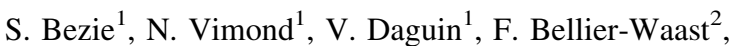
F. Duteille ${ }^{2}$, M. Levings ${ }^{3}$, B. Charreau ${ }^{1}$, I. Anegon ${ }^{1}$, C. Guillonneau ${ }^{1}$

${ }^{1}$ Inserm UMR1064 - CRTI, ITUN-CHU Nantes, Nantes, France ${ }^{2}$ Chirurgie Plastique Reconstructrice et Esthétique, $\mathrm{CHU}$ Nantes, Nantes, France ${ }^{3}$ University of British Columbiaand Child and Family Research Institute, Department of Surgery, Vancouver, Canada

Introduction: Regulatory $\mathrm{T}$ cell based immunotherapy is a promising treatment to prevent allograft from rejection. We previously demonstrated the therapeutic potential of polyclonal CD8 ${ }^{+}$Tregs in humanized mice transplantation models (Bézie, Front Immunol, 2018). Conferring donor-antigen specificity through chimeric antigen receptor (CAR) increased therapeutic potential of $\mathrm{CD}^{+}{ }^{+}$Tregs in mice models, suggesting lower dose requirement for treating patients and reduced risk of non-specific immunosuppression (MacDonald, JCI, 2017). This study aims to assess the potential of donor-antigen specific CD8 ${ }^{+}$Treg based therapy in transplantation.

Materials and Methods: Tregs were sorted on $\mathrm{CD} 8{ }^{+} \mathrm{CD}$ $45 \mathrm{RC}^{\text {low }}$ expression markers and expanded with anti-CD3/ CD28 mAbs in presence of IL-2 and IL-15. HLA-A2-CAR and irrelevant-CAR plasmids were kindly provided by $\mathrm{M}$. Levings to generate lentiviruses. Suppressive function was assessed on $\mathrm{CD} 4^{+} \mathrm{CD} 25^{-} \mathrm{T}$ cells stimulated by allogeneic APCs, one or the other expressing the HLA-A2 target. NSG mice were infused with HLA-A $2^{+}$PBMCs for xenogeneic GVHD model, or with HLA-A2 ${ }^{-}$BBMCs and were grafted with HLA-A2 ${ }^{+}$human skin for allograft model. 
Results: We demonstrated that $\mathrm{CD} 8^{+}$Tregs can be efficiently transduced and stably express A2-CAR, and 100-fold expanded in 14 days while maintaining a Tregs phenotype. Specific interaction with HLA-A $2^{+}$target cells activated A2-CAR CD ${ }^{+}$Tregs through zap-70 phosphorylation, upregulating activation markers and suppressive cytokines such as CD69, CD25, IL-34 and IFNgamma. Interestingly, calcium flux imaging revealed different activation kinetics through the CAR in Tregs vs Teffs. Importantly, A2-CAR CD ${ }^{+}$Tregs displayed minimal cytotoxic activity toward HLA-A2 ${ }^{+}$donor kidney graft endothelial cells after specific contact. Finally, A2-CAR $\mathrm{CD} 8^{+}$Tregs were more potent than irrelevant-CAR $\mathrm{CD} 8^{+}$Tregs to suppress HLA-A2 specific proliferation in vitro, and in vivo to prevent GVHD development induced by $\mathrm{HLAA} 2^{+} \mathrm{PBMCs}$ and to delay HLA-A2 ${ }^{+}$skin graft rejection in humanized mice models.

Conclusions: We have provided a proof of concept for clinical application in a MHC-I incompatible transplantation context, that the CAR CD8 ${ }^{+}$Treg-based therapy had high potential to prevent graft from rejection.

\section{ICLE8-0052}

Target and receptor discovery

\section{HIGHLY EFFICIENT AND SELECTIVE CAR-GENE TRANSFER USING CD4- AND CD8-TARGETED LENTIVIRAL VECTORS}

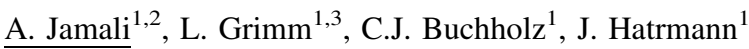

${ }^{1}$ Paul Ehrlich Institut, Molecular Biotechnology and Gene Therapy, Langen, Germany ${ }^{2}$ Faculty of Advanced Technologies in Medicine-Iran University of Medical Sciences, Molecular Medicine, Tehran, Iran ${ }^{3}$ German Cancer Consortium DKTK, Molecular Biotechnology and Gene Therapy, Heidelberg, Germany

Cellular immunotherapy has found exciting growth with CAR-T cells as a living drug which is an excellent example for the translation of basic science to the clinic. Lentiviral vectors (LVs) are one of the most applied vehicles for CAR gene delivery. Receptor-targeted LVs, such as CD4-LV and CD8-LV, delivering genes selectively to $\mathrm{T}$ cell subtypes may facilitate and improve CAR T cell generation but so far result in lower gene delivery rates than conventional LVs (VSV-LV). We studied the effect of the transduction enhancer Vectofusin-1 on gene delivery to human T cells with CD4- and CD8-targeted LVs encoding a second generation CD19-CAR and $\triangle$ LNGFR as reporter.

Vectofusin-1 enhanced the transduction rate up to $74 \%$ for CD4-LV and $80 \%$ for CD8-LV on average $(n=29$ or 34 ; determined at day 12 after transduction). In some samples transduction rates even approached $100 \%$, while retaining target cell selectivity. Interestingly, transduction rates of VSV-LV were lower in presence of Vectofusin-1 $(23-38 \%, \mathrm{n}=16)$ than in $\mathrm{ab}$ sence (52-73\%). Vectofusin-1 did not impair the killing capability of the generated CAR-T cells. Interestingly, a transient offtarget signal for $\triangle \mathrm{LNGFR}$ in samples treated with Vectofusin-1 was observed early after transduction. Increased washing steps during the transduction procedure resulted in decreased off-target positivity. To assess if this signal was caused by protein transfer, we incubated activated PBMC for 1 hour at $4^{\circ} \mathrm{C}$ with CD4-LV or CD8-LV. Notably, $\triangle$ LNGFR was readily detectable on targetreceptor positive cells as well as off-target cells by flow cytometry even in absence of Vectofusin-1. ELISA assays confirmed the presence of $\triangle \mathrm{LNGFR}$ in vector particles.

The data demonstrate that Vectofusin-1 can substantially raise gene delivery rates with CD4-LV and CD8-LV targeted vectors. This increase in gene delivery comes without affecting target cell selectivity and functionality, although a transient protein delivery to off-target cells can occur.

\section{ICLE8-0049}

Target and receptor discovery

\section{DIMERISATION IS ESSENTIAL FOR HIGH LEVEL CYTOKINE SECRETION BY T CELLS BEARING A CD28-BASED 2ND GENERATION CAR: POTENTIAL RELEVANCE FOR CONTROLLING CYTOKINE RELEASE SYNDROME}

\author{
A. Kilgallon ${ }^{1}$, M. Kalaitsidou ${ }^{1}$, G. Kueberuwa ${ }^{1}$, A. Schutt ${ }^{1}$, \\ D.E. Gilham $^{1}$, R.E. Hawkins ${ }^{1}$ \\ ${ }^{1}$ University of Manchester, Division of Cancer Sciences, \\ Manchester, United Kingdom
}

Most Chimeric Antigen Receptors (CARs) are dimeric molecules that generate down-stream signals through engineered activation and co-stimulatory cytoplasmic domains. To date, there are few general rules relating to the structural requirements for the activity of any specific CAR. We focused upon the role that dimerisation may play in CAR mediated signalling. CD28 based $2^{\text {nd }}$ generation CARs dimerise through specific disulphide bonds. We generated mutant CD28-based CARs that failed to dimerise and existed as monomers on the $\mathrm{T}$ cell surface. $\mathrm{T}$ cells bearing these monomeric CD28 CARs possessed an equivalent in vitro cytotoxicity capacity as compared to non-mutated dimerizing CD28 CARs. However, the monomeric CARs showed less lymphoproliferative and cytotoxic cytokine production capacity upon antigen challenge. Since CAR T cell therapy is commonly associated with severe cytokine release syndrome (CRS), the reduced level of cytokine production by monomeric CARs coupled with their high cytotoxic capacity warrant further in vivo investigation. This will determine whether the anti-tumour activity is maintained and associated with a reduced level of CRS. 